ARTICLE

\title{
Structural characterization of antibiotic self-immunity tRNA synthetase in plant tumour biocontrol agent
}

\author{
Shaileja Chopra ${ }^{1, *}$, Andrés Palencia ${ }^{2, *, \dagger}$, Cornelia Virus $^{1}$, Sarah Schulwitz ${ }^{1}$, Brenda R. Temple $^{3}$,
} Stephen Cusack $^{2} \&$ John Reader ${ }^{1, \dagger}$

Antibiotic-producing microbes evolved self-resistance mechanisms to avoid suicide. The biocontrol Agrobacterium radiobacter K84 secretes the Trojan Horse antibiotic agrocin 84 that is selectively transported into the plant pathogen $A$. tumefaciens and processed into the toxin TM84. We previously showed that TM84 employs a unique tRNA-dependent mechanism to inhibit leucyl-tRNA synthetase (LeuRS), while the TM84-producer prevents self-poisoning by expressing a resistant LeuRS AgnB2. We now identify a mechanism by which the antibiotic-producing microbe resists its own toxin. Using a combination of structural, biochemical and biophysical approaches, we show that AgnB2 evolved structural changes so as to resist the antibiotic by eliminating the tRNA-dependence of TM84 binding. Mutagenesis of key resistance determinants results in mutants adopting an antibiotic-sensitive phenotype. This study illuminates the evolution of resistance in self-immunity genes and provides mechanistic insights into a fascinating tRNA-dependent antibiotic with applications for the development of anti-infectives and the prevention of biocontrol emasculation.

\footnotetext{
${ }^{1}$ Department of Cell Biology and Physiology, The University of North Carolina at Chapel Hill, Chapel Hill, North Carolina 27599-7090, USA. ${ }^{2}$ European Molecular Biology Laboratory, Grenoble Outstation and Unit of Virus Host-Cell Interactions, UJF-EMBL-CNRS, UMI 3265, 71, Avenue des Martyrs, CS 90181 , 38042 Grenoble Cedex 96 rue Jules Horowitz, BP181, Grenoble Cedex 9 38042, France. ${ }^{3}$ R.L. Juliano Structural Bioinformatics Core Facility, The University of North Carolina at Chapel Hill, Chapel Hill, North Carolina 27599, USA. ${ }^{\star}$ These authors contributed equally to this work. † Present address: Institute for Advanced Biosciences, Team Host-Pathogen Interactions and Immunity to Infection, INSERM U1209, CNRS UMR5309, Université Grenoble Alpes, Grenoble Cedex 9 38042, France (A.P.); The Wellcome Trust Centre for Human Genetics, University of Oxford, Roosevelt Drive, Oxford OX3 7BN, UK (J.R.). Correspondence and requests for materials should be addressed to J.R. (email: jreader@well.ox.ac.uk)
} 
$\mathrm{M}$ icrobes that produce antimicrobial agents often prevent cell suicide by co-expressing self-immunity proteins that employ a variety of different resistance mechanisms ${ }^{1}$. Genes encoding self-immunity proteins can also act as a source of antibiotic resistance in the environment when they are horizontally transferred to microbial pathogens ${ }^{2}$. This threatens not only the successful treatment of human pathogens but also prevention of important animal and crop diseases ${ }^{3}$. One mechanism of self-immunity is to encode resistant variants of the enzyme targeted by the antibiotic. A fascinating example of such an antibiotic-producing organism is Agrobacterium radiobacter strain K84, which is used as a biocontrol agent of the plant pathogen A. tumefaciens that causes plant tumours in a number of agriculturally important species ${ }^{4-6}$. The biocontrol produces the 'Trojan Horse' antibiotic agrocin 84 (refs 7-9) that mimics a plant tumour-derived substrate to gain entry by subterfuge into the pathogen ${ }^{10,11}$, where it is processed into a toxin termed TM84 (refs 10,12) (Figs 1 and 2a). We recently identified the molecular target of TM84 in A. tumefaciens as leucyl-tRNA synthetase $\left(\right.$ LeuRS $_{\mathrm{At}}$ ) which catalyses an essential first step in protein synthesis ${ }^{12}$. To prevent cell suicide, A. radiobacter K84 contains a second, non-essential LeuRS, termed AgnB2, which is resistant to TM84 (refs 12-14) (Fig. 1). The agnB2 gene is encoded on the $44 \mathrm{~kb}$ plasmid pAgK84, along with all the genes required to biosynthesize agrocin 84 (refs 14,15). Importantly, horizontal gene transfer of the pAgK84 plasmid from A. radiobacter $\mathrm{K} 84$ to the pathogen has been previously reported to occur in the field, resulting in agrocin 84-resistant strains of $A$. tumefaciens encoding the agnB2 gene $5,16,17$. Thus, understanding the molecular mechanisms by which AgnB2 provides immunity to agrocin 84 is crucial for combating antibiotic resistance.

The molecular target of TM84, LeuRS $\mathrm{At}_{\text {t }}$ is an essential enzyme that specifically ligates leucine to its cognate tRNA ${ }^{\text {Leu }}$ isoacceptors, a critical step before protein synthesis at the ribosome. The aminoacylation of tRNA ${ }^{\text {Leu }}$ is catalysed in a two-step mechanism in all LeuRSs: in the tRNA-independent first step, leucine is activated by ATP to form the essential obligate intermediate leucyl-adenylate (Leu-AMP) that is stably bound to the enzyme. In the second step, a bound tRNA ${ }^{\mathrm{Leu}}$ repositions its CCA $3^{\prime}$-end into the LeuRS active site where activated-leucine is transferred to the tRNA. The essential catalytic nature of LeuRSs, and the 19 other canonical aminoacyl-tRNA synthetases (AARSs), has led to these enzymes emerging as prominent targets for anti-pathogens ${ }^{18,19}$, ranging in the prevention of fungal diseases in humans ${ }^{20}$ to bacterial plant pathogens ${ }^{12}$. A substantial amount of work has been performed on stable analogues of aminoacyl-adenylates that bind tightly to an AARS active site, and preventing activation of the amino acid with ATP $^{21,22}$. However, these molecules often cannot be transported efficiently into cells and can lack discrimination between microorganism and the intended host ${ }^{18}$.

We recently showed that TM84 does not behave like other stable aminoacyl-adenylate analogues but, instead, employs a unique tRNA-dependent inhibition mechanism ${ }^{23,24}$. TM84 binds only weakly to the LeuRS-active site on its own and requires the presence of tRNA ${ }^{\text {Leu }}$ to form a tight binding ternary inhibition complex ${ }^{23}$. The X-ray crystal structure of the Escherichia coli LeuRS $_{\mathrm{Ec}} \bullet \mathrm{tRNA}{ }^{\mathrm{Leu}} \bullet$ TM84 ternary complex shows TM84 bound in the Leu-AMP-binding site of the catalytic domain. The tRNA ${ }^{\mathrm{Leu}}$ is bound to LeuRS in an 'aminoacylation-like' conformation with the CCA $3^{\prime}$-end of the tRNA ${ }^{\text {Leu }}$ entering into the active site with the terminal adenosine hydrogen bonding (H-bonding) directly with TM84 (ref. 23). The positioning of the tRNA ${ }^{\text {Leu }}$ acceptor stem is accompanied with a substantial movement of the catalytic $\mathrm{K}^{619} \mathrm{MSK}^{622} \mathrm{~S}$ motif. The $\mathrm{K} 619$ sidechain bonds to the penultimate tRNA ${ }^{\text {Leu }}$ cytidine base, while K622 extends across the adenylate analogue and interacts with the phosphate of TM84 via a non-bridging oxygen. The repositioning of the Lys-Met-SerLys-Ser (KMSKS) loop and tRNA ${ }^{\text {Leu }}$ combine to effectively bury the bound TM84, thus explaining how the presence of tRNA ${ }^{\mathrm{Leu}}$ induces the tight binding of LeuRSs to TM84.

AgnB2 exhibits a high degree of resistance to TM84 (ref. 12) despite the close similarity of TM84 to Leu-AMP (Fig. 2a) and the

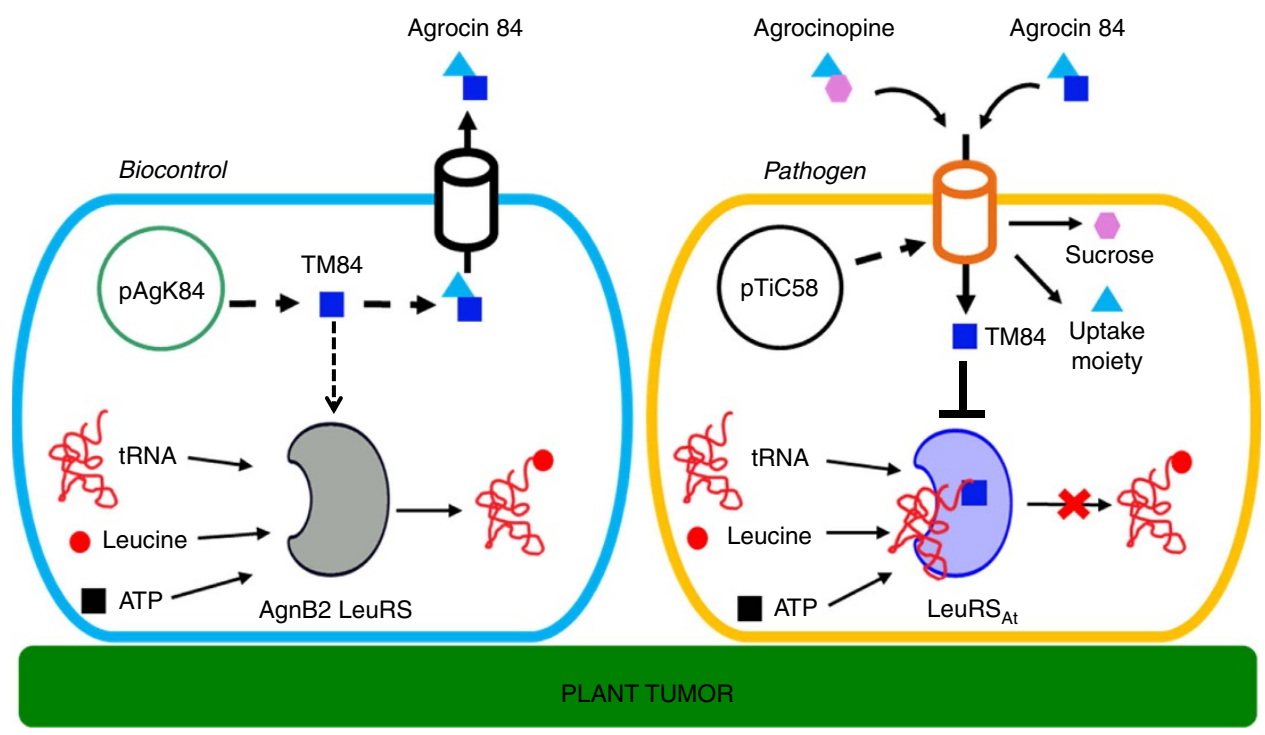

Figure 1 | Biology of agrocin 84 produced by biocontrol A. radiobacter strain K84. Pathogenic A. tumefaciens possesses a TiC58 plasmid that upon infection of the host plant makes the plant produce a carbon and phosphate source agrocinopine. The pathogen takes up agrocinopine via an agrocinopine permease encoded on pTiC58. This transporter also recognizes the uptake moiety on agrocin 84 , an antibiotic produced by the plant tumour biocontrol, A. radiobacter strain $\mathrm{K} 84$. Upon entry of agrocin 84 into the pathogen, it is cleaved into the toxic moiety TM84 and the transport moiety. TM84 targets

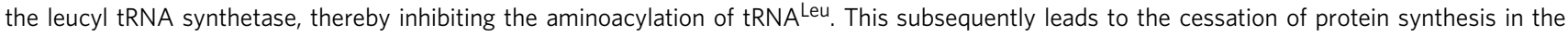
pathogen and leads to cell death. TM84 however has no effect on the aminoacylation reaction of a self-immunity LeuRS called AgnB2 that is encoded by the pAgK84 plasmid in A. radiobacter K84. 

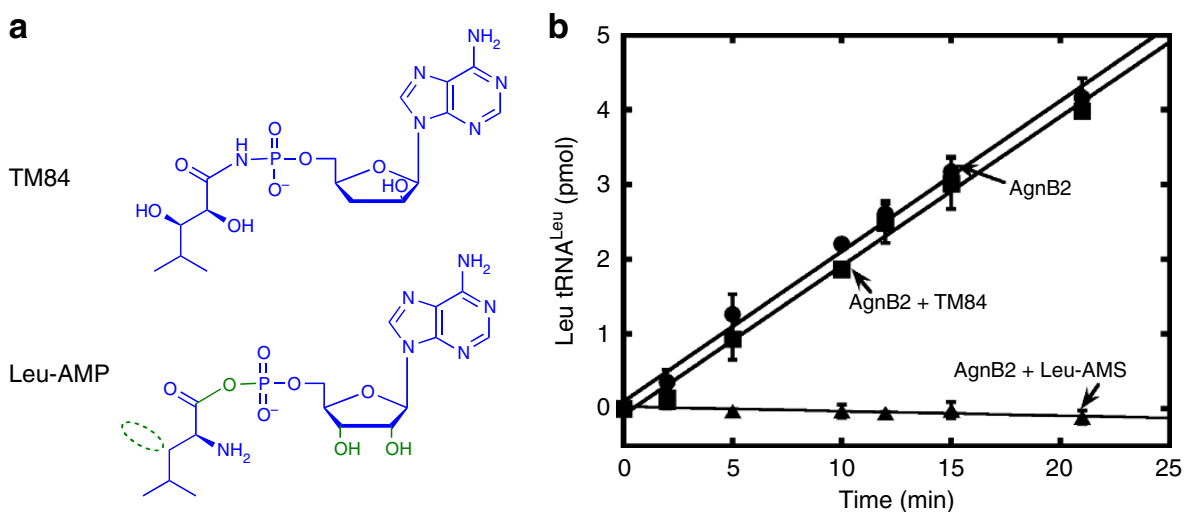

Figure 2 | AgnB2 discriminates between a stable Leu-AMP analogue and TM84. (a) Chemical structures of Leu-AMP and TM84 with differences highlighted in green. A non-hydrolysable adenylate analogue called Leu-AMS, which contains a N-sulfamoyl linkage in place of the phosphoanhydride of Leu-AMP, was used in biophysical and crystallography experiments. (b) Effect of TM84 and Leu-AMS on the aminoacylation reaction of wt AgnB2.

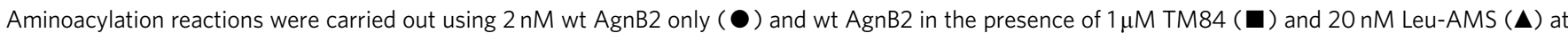
$28^{\circ} \mathrm{C}, \mathrm{pH} 7.4$ and initiated using $1 \mathrm{mM}$ ATP. Error bars represent s.d. $(n=3)$.

need for AgnB2 to adopt a true aminoacylation complex as exhibited in a recent LeuRS $_{\mathrm{Ec}} \bullet \mathrm{tRNA}^{\mathrm{Leu}} \bullet$ Leu-AMS crystal structure $^{25}$. These observations raise the question of how does AgnB2 achieve resistance to TM84 without dramatically interfering with the catalysis of the aminoacylation reaction. This is particularly intriguing as primary sequence alignments of AgnB2 and TM84-sensitive LeuRSs show a high similarity of residues, including catalytic motifs (except a single Q instead of $\mathrm{M}$ at the KMSKS motif). Here, we examine the TM84 resistance mechanisms employed by AgnB2 using both thermodynamics and steady-state enzyme kinetics. We also present the X-ray crystal structure of AgnB2 in complex with tRNA ${ }^{\text {Leu }}$ and a stable leucyl-adenylate analogue and reveal tertiary interactions unique to AgnB2. We finally identify key TM84 immunity determinants

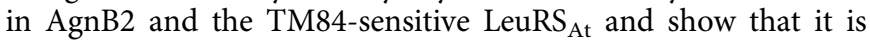
possible to partially inter-convert the TM84 sensitivity properties of these enzymes.

\section{Results}

Analysis of AgnB2 steady state enzyme kinetics. The ability of AgnB2 to discriminate between TM84 and the obligate reaction intermediate Leu-AMP prompted us to examine differences between the two molecules. As shown in Fig. 2a, TM84 contains a stable $\mathrm{N}$-acyl phosphoamidate bond, instead of the reactive phosphoanhydride linkage in Leu-AMP. In addition, TM84 has a deoxy-arabinose sugar group, instead of a ribose with subsequent loss of a $3^{\prime}$-hydroxyl $(\mathrm{OH})$ and a repositioned $2^{\prime}-\mathrm{OH}$ above the sugar ring, and replacement of the primary amine with another $\mathrm{OH}$ group. For TM84-sensitive LeuRSs, these subtle differences between toxin and reaction intermediate allow TM84 to employ a tRNA-dependent inhibition mechanism where the CCA $3^{\prime}$-end of the tRNA ${ }^{\text {Leu }}$ binds in an 'aminoacylation-like conformation' into the active site of the enzyme and directly hydrogen bonds with the antibiotic ${ }^{23}$. The 'aminoacylation-like' conformation of tRNA in the LeuRS $_{\mathrm{Ec}} \bullet \mathrm{tRNA}^{\text {Leu }} \bullet$ TM84 ternary inhibition complex exhibits a high degree of similarity with the $\mathrm{tRNA}^{\text {Leu }}$ in the true LeuRS aminoacylation conformation ${ }^{25}$. Since the tRNA ${ }^{\text {Leu }}$ bound to AgnB2 still has to reposition its CCA $3^{\prime}$-end into the active site during catalysis of the aminoacyl transfer reaction, we questioned whether destabilization of the TM84 ternary complex in AgnB2 might be reflected in the kinetic properties of AgnB2.

Our steady-state kinetic analysis of AgnB2 examined both the overall aminoacylation reaction and the first step, the amino acid activation reaction, using the $\mathrm{ATP} / \mathrm{PPi}$ exchange reaction. We firstly determined the apparent TM84 inhibition constant
Table 1 | Kinetic parameters obtained for the aminoacylation and ATP-PPi exchange reactions catalysed by LeuRS ${ }_{\mathrm{At}}$ and AgnB2 at $28^{\circ} \mathrm{C}$ at $\mathrm{pH} 7.4$.

\begin{tabular}{|c|c|c|c|}
\hline Enzyme & Substrate & $K_{\mathrm{m}}(\mu \mathrm{M})$ & $k_{\text {cat }}\left(s^{-1}\right)$ \\
\hline \multicolumn{4}{|c|}{ Aminoacylation } \\
\hline \multirow[t]{3}{*}{ AgnB2 } & ATP & $95.4 \pm 18.3$ & $0.43 \pm 0.02$ \\
\hline & Leucine & $28.7 \pm 7.3$ & $0.35 \pm 0.03$ \\
\hline & tRNA & $1.48 \pm 0.25$ & $0.43 \pm 0.02$ \\
\hline \multirow[t]{3}{*}{ LeuRS $_{A T}{ }^{\star}$} & ATP & $396 \pm 83$ & $0.33 \pm 0.02$ \\
\hline & Leucine & $38 \pm 3.2$ & $0.38 \pm 0.01$ \\
\hline & tRNA & $0.94 \pm 0.19$ & $0.36 \pm 0.02$ \\
\hline \multicolumn{4}{|c|}{ ATP-PP ${ }_{i}$ Exchange } \\
\hline \multirow[t]{2}{*}{ AgnB2 } & ATP & $1047 \pm 140$ & $67 \pm 8.9$ \\
\hline & Leucine & $12 \pm 2$ & $73 \pm 3.6$ \\
\hline \multirow[t]{2}{*}{ LeuRS $_{\text {AT }}$} & ATP & $357 \pm 22$ & $87 \pm 1.5$ \\
\hline & Leucine & $9.7 \pm 0.7$ & $85 \pm 1.6$ \\
\hline \multicolumn{4}{|c|}{$\begin{array}{l}\text { All data in this table were average values obtained from three independent experiments with } \\
\text { error } \pm \text { values representing s.d. } \\
{ }^{*} \text { From reference Chopra, et al. }{ }^{23} \text {. }\end{array}$} \\
\hline
\end{tabular}

$\left(K_{i}\right)$ for AgnB2 in the aminoacylation reaction by altering the ATP concentrations of the substrate and pre-incubating the AgnB2॰tRNA ${ }^{\text {Leu }}$ complex before initiating the reaction. The data revealed an apparent $K_{i}$ of $4.72 \pm 0.75 \mu \mathrm{M}$ (Supplementary Fig. 1). We then determined the apparent TM84 $K_{i}$ for AgnB2 by altering the leucine concentration and keeping the ATP fixed giving a value of $1.38 \pm 0.28 \mu \mathrm{M}$ (Supplementary Fig. 1a,b). Both values are more than three orders of magnitude weaker than the $K_{i}$ app determined previously for the natural target LeuRS ${ }_{\text {At }}$ (ref. 23). Importantly, TM84 acted as a weak competitive inhibitor of AgnB2 with regards to both ATP and leucine (Supplementary Fig. 1a,b, respectively) and therefore still binds to the synthetic active site and not at an alternative site. We also found that AgnB2 is resistant to TM84, but is still potently inhibited by Leu-AMS (a non-hydrolysable analogue of the Leu-AMP reaction intermediate), for both the amino acid activation (Supplementary Fig. 2) and overall aminoacylation reactions (Fig. 2b). Substantial weakening of the binding of the aminoacyl adenylate to AgnB2 did not evolve as a solution to generating resistance to TM84, as this would severely affect the catalysis of amino acid activation.

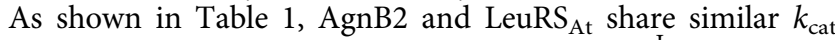
values for all substrates. The higher $K_{\mathrm{m}}$ for tRNA ${ }^{\text {Leu }}$ in AgnB2 may reflect an alteration in the interactions of AgnB2 with 
tRNA $^{\text {Leu }}$ that is relevant to resistance to the tRNA-dependent TM84 inhibitor. The $K_{\mathrm{m}}$ for ATP is a small 3.8-fold higher affinity value for AgnB2 than for LeuRS ${ }_{\mathrm{At}}$, while the leucine affinities show minimal differences. In the case of ATP/PPi exchange reaction, AgnB2 showed a higher $K_{\mathrm{m}}$ for ATP compared with the aminoacylation reaction (10-fold increase). Interestingly, these kinetic comparisons represent only small energetic differences between the two enzymes, yet still lead to enzymes with pronounced differences in TM84 sensitivity. In addition, the steady-state kinetic properties shows that the ATP saturation plot for the overall aminoacylation reaction shows a degree of substrate inhibition, whereas the ATP/PPi exchange shows no substrate inhibition for ATP (data not shown) when tRNA $^{\text {Leu }}$ is absent. One possibility is the population of a second unproductive ATP-binding site in the AgnB2 active site that may in part explain the differences between the apparent TM84 $K_{i}^{\text {'s }}$ determined for AgnB2 when varying leucine and ATP (Supplementary Fig. 1). Interestingly, we do not observe any ATP substrate inhibition for LeuRS $_{\text {At }}$. The data suggest that different substrate-dependent conformations populated by AgnB2 may be important for TM84 resistance.

tRNA ${ }^{\text {Leu }}$ has reduced effect on the affinity of TM84 to AgnB2. If resistance of AgnB2 is based on abolishing of the normal tRNA-dependent inhibition mechanism employed by TM84 (ref. 23) then one would expect to see a decrease in the affinity for TM84, tRNA ${ }^{\text {Leu }}$ and/or for TM84 in the presence of tRNA or vice versa. To test these possibilities, we used isothermal calorimetry (ITC) (Supplementary Table 1) to investigate the ligand binding modes to AgnB2 (ref. 23). $K_{\mathrm{d}}$ values of $1052 \mathrm{nM}$ for TM84 and $310 \mathrm{nM}$ for tRNA were obtained, indicating reductions in AgnB2binding affinities for both TM84 (6.9-fold) and tRNA (3.7-fold), compared with LeuRS At (ref. 23). Further examination of TM84 binding to AgnB2 (with pre-bound tRNA) showed a $K_{\mathrm{d}}$ of $332 \mathrm{nM}$, which suggests a substantial destabilization of

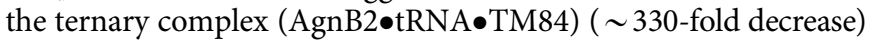
as compared with the LeuRS $\mathrm{At} \bullet \mathrm{tRNA} \bullet \mathrm{TM} 84$ complex (Supplementary Table 2). Interestingly, we also see no evidence of a binary isotherm for TM84 binding to AgnB2॰tRNA ${ }^{\text {Leu }}$

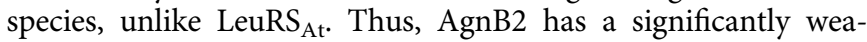

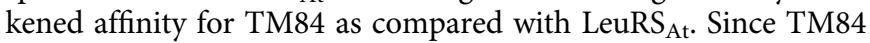
and the Leu-AMP analogue bind to the same active site but potentially to different conformations, we examined if AgnB2binding affinity for Leu-AMS had been substantially weakened. Our results show that Leu-AMS binds to AgnB2 with low nanomolar binding affinity $\left(K_{\mathrm{d}}=4.4 \mathrm{nM}\right)$, which is slightly weaker than to LeuRS $(1.6 \mathrm{nM})$. The fact that AgnB2 binds tightly to Leu-AMS is not so surprising since, firstly, AgnB2 has highly conserved residues at the synthetic site compared with other LeuRSs and, secondly, the enzyme must bind tightly to the Leu-AMP intermediate to catalyze the aminoacylation reaction. This result was supported by our enzyme kinetic observations above which showed that Leu-AMS is a low nanomolar inhibitor of both the AgnB2-catalyzed aminoacylation and amino acid activation reactions, demonstrating that the analogue efficiently competes with ATP and leucine substrates (Supplementary Fig. 1).

Our thermodynamic results suggest that AgnB2 may achieve resistance to TM84 by not simply weakening the affinity for TM84 (and/or tRNA ${ }^{\text {Leu }}$ ) but most significantly by destabilizing the formation of the AgnB2॰tRNA ${ }^{\mathrm{Leu}} \bullet$ TM84 complex. Interestingly, this is accomplished with only minimal change to affinity for the adenylate, allowing the enzyme to still efficiently carry out aminoacylation.

Structure of AgnB2॰tRNA ${ }^{\text {Leu }} \bullet$ Leu-AMS ternary complex. To identify structural interactions in AgnB2 that may contribute to resistance, we performed extensive crystallizations trials with AgnB2, agrobacterium tRNA ${ }^{\text {Leu }}$ and multiple combinations of substrates or inhibitors. We obtained an X-ray crystal structure of AgnB2 in complex with tRNA ${ }^{\text {Leu }}$ and Leu-AMS; the final model was refined to a final R-factor of $19.4 \%$ (R-free $=23.8 \%$ ) and a final resolution of $2.1 \AA$ (Table 2, Fig. 3a and Supplementary Fig. 3).

The overall AgnB2॰tRNA ${ }^{\text {Leu }} \bullet$ Leu-AMS structure (Fig. 3a, and see domain architecture in Fig. 4) presents a similar conformation to a number of bacterial LeuRS structures obtained in complex with tRNAs ${ }^{\text {Leu, }}$ in which the CCA $3^{\prime}$-end is positioned into the editing active site and represents what appears to be the preferred LeuRS conformation ${ }^{20,25,26}$. This editing conformation of the tRNA in the AgnB2 complex is in contrast to the structures of the TM84 ternary inhibition complex of

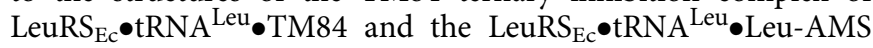
aminoacylation complex which have the CCA $3^{\prime}$-end penetrating into the catalytic active site of the enzyme positioned next to the bound inhibitors ${ }^{23,25}$. Another important interaction observed in the structure is the C-terminal domain interaction with the variable loop of Agrobacterium tRNA ${ }^{\text {Leu }}$ (Supplementary Fig. 4a). A comparison of the aminoacylation and editing states in LeuRS $_{\mathrm{Ec}}$, shows the C-term domain coupled to the variable arm of $E$. coli tRNA ${ }^{\text {Leu }}$ rotates significantly upwards towards the catalytic domain in the aminoacylation state. It has been hypothesized that this C-term domain movement propagates towards the main body of the tRNA ${ }^{\text {Leu }}$ and towards the acceptor stem, and together with rotations of other external domains, or residues, forces the $3^{\prime}$-end of the tRNA into the catalytic site.

Table 2 | Data collection and refinement statistics

\begin{tabular}{|c|c|}
\hline \multicolumn{2}{|c|}{$\begin{array}{l}\text { Agrobacterium radiobacter strain K84 } \\
\text { AgnB2 LeuRS + tRNA }{ }^{\text {Leu }}+\text { Leu-AMS }\end{array}$} \\
\hline \multicolumn{2}{|l|}{ Data collection } \\
\hline Space group & $P 2_{1}$ \\
\hline \multicolumn{2}{|l|}{ Cell dimensions } \\
\hline$a, b, c(\AA)$ & $169.91,50.32,170.52$ \\
\hline$\alpha, \beta, \gamma\left({ }^{\circ}\right)$ & $90.00,93.48,90.00$ \\
\hline Resolution $(\AA)^{\star}$ & $50-2.1(2.10-2.20)$ \\
\hline$R_{\mathrm{sym}}$ & $7.5(65.2)$ \\
\hline$|/ \sigma|$ & $10.6(2.0)$ \\
\hline Completeness (\%) & $99.5(99.8)$ \\
\hline Redundancy & $3.7(3.7)$ \\
\hline \multicolumn{2}{|l|}{ Refinement } \\
\hline Resolution $(\AA)$ & $48.6-2.1(2.15-2.10)$ \\
\hline No. of reflections work/free & $160,330 / 8,487$ \\
\hline$R_{\text {work }} / R_{\text {free }}$ & $0.194(0.309) / 0.237(0.331)$ \\
\hline \multicolumn{2}{|l|}{ No. of atoms } \\
\hline Protein & {$[6,307]^{A},[6,141]^{B}$} \\
\hline tRNA ${ }^{\text {Leu }}$ & {$[1,702]^{C},[1,721]^{\mathrm{D}}$} \\
\hline Ligand & 62 [2 × Leu-AMS] \\
\hline Water $/ \mathrm{SO}_{4}^{-2}$ & $871 / 25\left[5 \times \mathrm{SO}_{4}^{-2}\right]$ \\
\hline $\mathrm{Mn}^{2+}$ & 2 \\
\hline \multicolumn{2}{|l|}{$B$-factors } \\
\hline Protein & {$[52.3]^{A},[48.6]^{B}$} \\
\hline tRNA ${ }^{\text {Leu }}$ & {$[57.7]^{\mathrm{C}},[54.9]^{\mathrm{D}}$} \\
\hline Ligand & {$[27.8]^{\mathrm{E}},[26.6]^{\mathrm{F}}$} \\
\hline Water $/ \mathrm{SO}_{4}^{-2}$ & $44.4 / 86.2$ \\
\hline $\mathrm{Mn}^{2+}$ & 87.3 \\
\hline \multicolumn{2}{|l|}{ RMS deviations } \\
\hline Bond lengths $(\AA)$ & 0.016 \\
\hline Bond angles $\left({ }^{\circ}\right)$ & 1.736 \\
\hline
\end{tabular}


Interestingly, the tRNA ${ }^{\text {Leu }}$ from Agrobacterium has a shorter variable stem than the $E$. coli tRNA, therefore questioning whether this propagation force due to coupling of tRNA with the
C-term domain is also required in AgnB2 for aminoacylation. In fact, a comparison of the C-terminal domain from AgnB2 compared with the $E$. coli editing structure $\left(\text { LeuRS }_{\mathrm{Ec}} \bullet \mathrm{tRNA}^{\mathrm{Leu}}\right)^{25}$

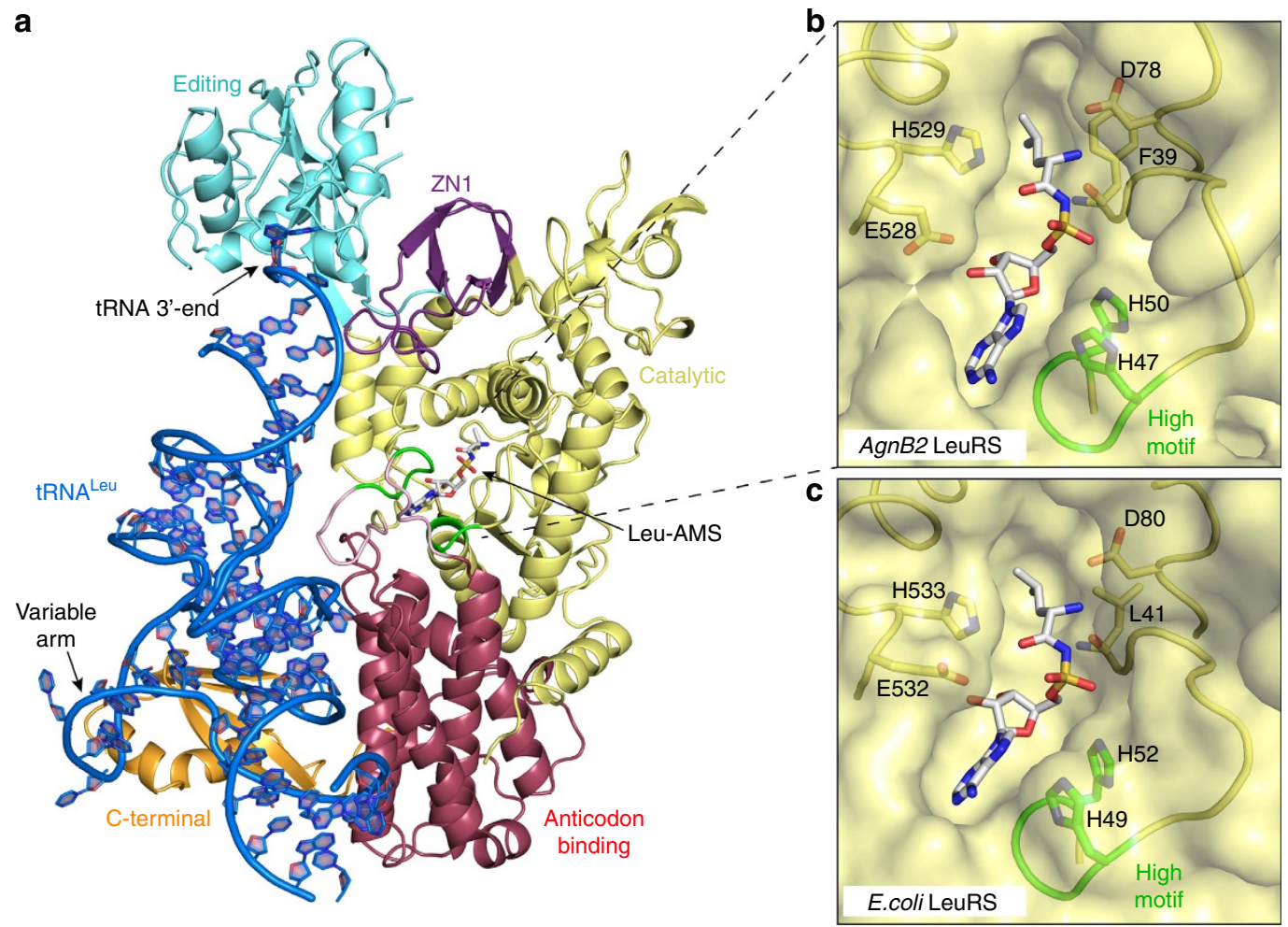

Figure 3 | Structure of the AgnB2॰tRNA ${ }^{\text {Leu } \bullet L e u-A M S ~ c o m p l e x . ~(a) ~ X-r a y ~ s t r u c t u r e ~ o f ~ w i l d-t y p e ~ A g n B 2 \bullet t R N A ~}{ }^{\text {Leu }} \bullet$ Leu-AMS complex with $3^{\prime}$-tRNA ${ }^{\text {Leu }}$ (blue) bound in the editing site (PDB Code-5AH5). The catalytic, editing, anticodon binding and C-terminal domains are displayed in yellow, cyan blue, red and gold, respectively. Leu-AMS bound to the synthetic active site is represented in a stick structure and the catalytic KQSKS and HIGH loops are highlighted in green. $(\mathbf{b}, \mathbf{c})$ Comparison of wt AgnB2 $\bullet$ RNA $\bullet$ Leu-AMS complex and LeuRS $\mathrm{Ec}_{\bullet} \bullet$ tRNA ${ }^{\text {Leu }} \bullet$ Leu-AMS complex binding site residues. The surface of the catalytic active site of the protein is depicted in yellow with Leu-AMS bound (stick structure).

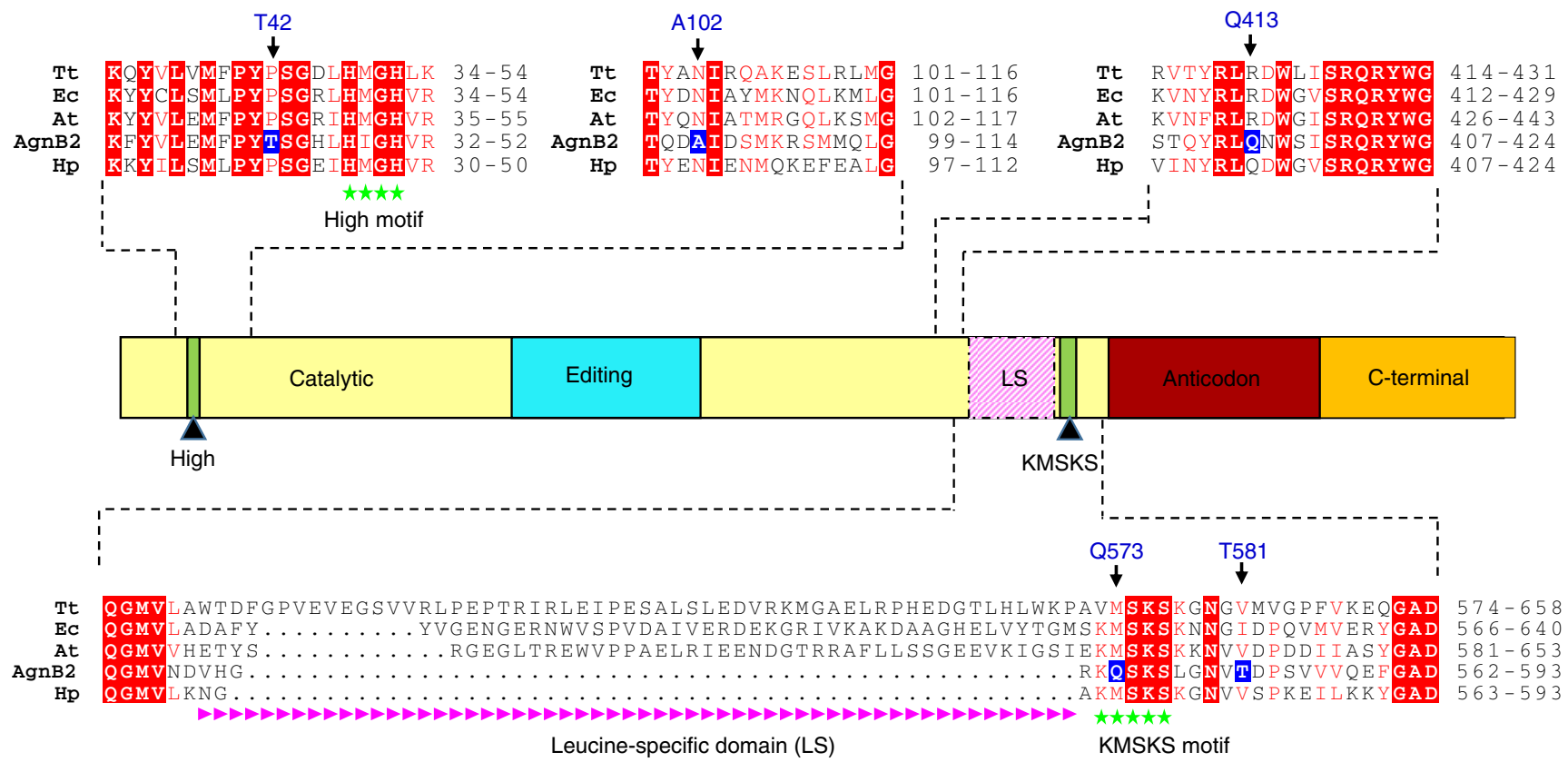

Figure 4 | Domain structure and primary sequence alignment of LeuRSs and AgnB2. Domain structure of LeuRS and primary sequence alignment of the catalytic domain and LS domains of Thermus thermophilus LeuRS (LeuRS $\mathrm{Tt}_{\mathrm{t}}$ ), LeuRS $\mathrm{Ec}_{\mathrm{E},}$ LeuRS $_{\mathrm{At}}$, plasmid encoded AgnB2 (from A. radiobacter strain K84) and LeuRS $\mathrm{Hp}_{\mathrm{H}}$. The highly conserved regions are depicted in grey and the residues highlighted in bold (blue) represent the non-conserved residues in AgnB2. In addition, both AgnB2 and LeuRS ${ }_{\mathrm{Hp}}$ lack the leucine-specific domain (pink) that is present in other LeuRSs. 
shows there is a rotation of about $18.5^{\circ}$ and the shorter variable loop of agrobacterium only makes one contact with the phosphate of nucleotide $46 \mathrm{f}$ (P46f) vs the E. coli tRNA which makes multiple contacts with the variable domain ${ }^{25}$ (Supplementary Fig. 4a,b). This structure may give new insights into the function of this stem-loop.

Unique amino acid substitutions in the AgnB2 catalytic site. Close inspection of the Leu-AMS-binding site in the AgnB2 structure reveals that the residues at the catalytic site are very similar compared with those found in LeuRS $\mathbf{E c}$ Fig. 3b,c. There are small differences including F39 in AgnB2, forming part of the hydrophobic pocket for the leucine, vs the corresponding L41 found in the LeuRS $S_{\mathrm{Ec}}$. Additionally, E528 in AgnB2 exhibits a different rotamer away from ribose hydroxyl vs the corresponding E532 residue in the LeuRS $\mathrm{Ec}_{\mathbf{E c}}$ aminoacylation complex, which is situated more closely to the ribose hydroxyl. The LeuRS $\mathrm{Ec}_{\mathbf{E}}$ E532 plays an important role in catalysis of the aminoacylation reaction, as suggested by the LeuRS $\mathrm{Ec}_{\bullet} \bullet \mathrm{tRNA}^{\mathrm{Leu}} \bullet$ Leu-AMS complex structure and confirmed by biochemical experiments ${ }^{25}$. The observation that TM84-sensitive LeuRS $_{\mathrm{Ec}}$ and resistant AgnB2 showed no substantial differences between active site residues directly interacting with Leu-AMS might explain how the two enzymes can bind equally well to Leu-AMS and carry out aminoacylation with close kinetic parameters (see above). It therefore follows that no AgnB2 residues are directly in a position to interrogate the $5^{\prime} \mathrm{N}$-acyl phosphoramidate bond of TM84 when it is bound to the enzyme alone. The weaker binding of

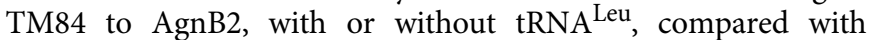

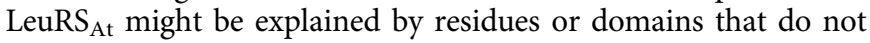
directly interact with the adenylate analogue, but which could promote long range effects or conformational changes affecting the catalytic site or perturbing the interaction with tRNA ${ }^{\text {Leu. }}$.

The KMSKS catalytic peptide motif, along with the HIGH loop, is present in all Class I aaRSs, including LeuRSs, and has been shown to play a critical role in the both the amino acid activation reaction and the aminoacyl transfer reactions ${ }^{27,28}$. Biochemical and biophysical studies on class I synthetases, including LeuRS, shows that ATP binding to the catalytic active site leads to a conformational change in the mobile KMSKS loop shifting it from an 'open' to a 'closed' conformation with the two lysine residues of the KMSKS loop interacting with the $\alpha$ and $\beta$ phosphates of the ATP ${ }^{27-29}$. Once the aminoacyl-adenylate is formed, the KMSKS loop moves into a 'semi-open' conformation to allow for the entry of the CCA $3^{\prime}$-end of the tRNA into the active site. Finally, once the CCA $3^{\prime}$-end of the tRNA is in the active site, the KMSKS loop once again adopts the closed conformation $^{25,27,30}$. In the case of AgnB2, the highly conserved methionine of the KMSKS motif is interestingly replaced by a glutamine residue (Q573). Our structure shows that Leu-AMS is bound in the active site with the mobile KQSKS loop present in a semi-open conformation (Supplementary Fig. 5). This loop position is in contrast to the fully closed position exhibited by the KMSKS loop in the LeuRS $\mathrm{Ec}_{\mathrm{E}} \bullet \mathrm{tRNA} \bullet \mathrm{TM} 84$ ternary complex where the second lysine of the KMSKS loop (K622) directly interacts with the non-bridging phosphate of the nucleotide analogue and the first lysine of the KMSKS loop (K619) interacts with cytosine 75 of the tRNA $3^{\prime}$-end ${ }^{23}$. In Class I aaRSs, this conserved methionine forms a number of hydrophobic packing interactions and is thought to play an important role in aminoacylation $^{31-34}$. In LeuRS structures it also forms a main chain interaction with the adenine ring of the bound adenylate $^{25,35}$. The equivalent position in AgnB2, Q573, forms a hydrogen bond side-chain interaction with the side chain of T581 (Fig. 5a), which is found to be a conserved hydrophobic residue in TM84 sensitive LeuRSs (I628 in LeuRS $\mathrm{Ec}_{\mathrm{c}}$ ) (Fig. 5b). It is interesting to note that the AgnB2 KQSKS loop exhibits a distinctly shifted position relative to the Leu-AMP binding pocket (Supplementary Fig. 5), perhaps due to these changes. The fact that a glutamine residue is found in this critical catalytic motif but not in any other LeuRS in nature, may indicate an important role for Q573 in TM84 resistance. It maybe that a substantial number of compensatory mutations are required to accommodate Q573 in AgnB2.

T42 and A102 are two other residues that are specific to AgnB2. T42 is positioned upstream from the catalytic $\mathrm{H}^{47} \mathrm{IGH}^{50}$ motif, while the side chain of A102 is situated in close proximity to T42 on a neighbouring $\alpha$-helix (Fig. 5). Importantly, the presence of A102 in AgnB2, instead of an asparagine as found in the LeuRS $\mathrm{Ec}_{\mathrm{Ec}}$ structure (N104), leads to loss of an H-bond interaction between the $\alpha$-helix (96-114) and a main-chain peptide bond located at T42 (P45 LeuRS $\mathrm{Ec})$. Both these modifications could allow for subtle changes in the positioning of the neighbouring HIGH motif that binds TM84. a

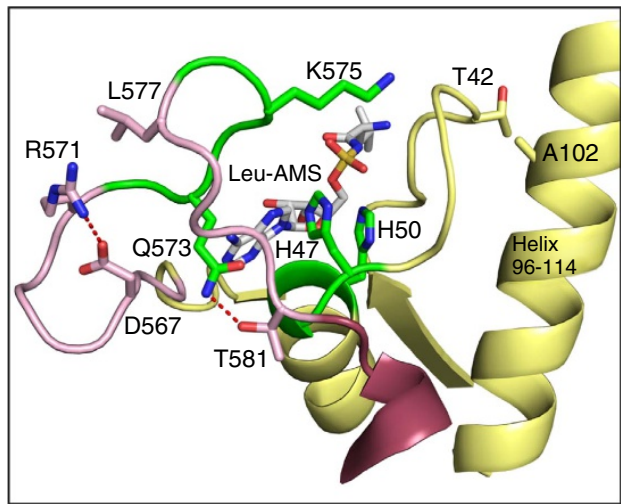

b

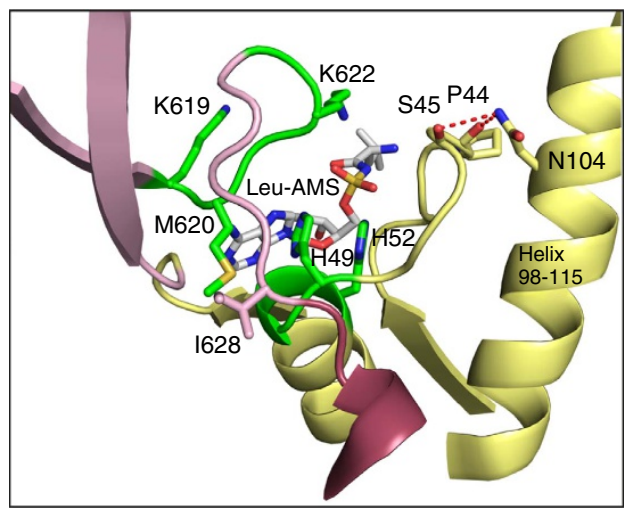

Figure 5 | Interactions of key residues in the catalytic active site of AgnB2. Ribbon structure of the catalytic active site of (a) AgnB2•tRNA ${ }^{\text {Leu }} \bullet$ Leu-AMS complex and (b) E. coliøtRNA ${ }^{\text {Leu }}$ Leu-AMS complex. AgnB2 key residues interacting with Leu-AMS (shown as white sticks) or adjacent to the catalytic site are depicted as sticks with the following colour code: residues of the $\mathrm{K}^{572} \mathrm{QSKS}{ }^{576}$ and of the $\mathrm{H}^{47} \mathrm{IGH}^{50}$ catalytic motifs, are coloured in green; core permissive residues T42 and A102 are shown in yellow; T581 which H-bonds to Q573 in pink; and D567 and R571 which forms the salt bridge mutated in this study and residue L577 are also in pink. E. coli LeuRS equivalent residues in the aminoacylation complex (PDB: 4AQ7) are shown in panel b. Key interactions in both the AgnB2 and the E. coli complexes are shown as red-dashed lines. 
AgnB2 has modified tRNA elements compared with LeuRS $S_{\mathrm{Ec}}$. A prominent feature of the AgnB2 structure is the lack of an idiosyncratic leucyl-specific insertion domain (LS-domain), which is present in bacterial LeuRS, except in a few cases such, as Bacillus subtilis or Mycoplasma mobile ${ }^{25,36}$, and as seen in the Helicobacter pylori LeuRS $\left(\right.$ LeuRS $_{\mathrm{Hp}}$ ) primary amino acid sequence in Fig. 4. The LS-domain undergoes substantial movement between the LeuRS $\mathrm{Ec}_{\mathrm{Ec}}$ editing, and the TM84 and Leu-AMS bound 'aminoacylation-like' conformations, buttressing the highly mobile KMSKS loop as well as guiding the tRNA into the catalytic site, as found in the aminoacylation conformation ${ }^{25}$. The missing LS-domain in AgnB2 is replaced by a small connecting loop that contains a salt bridge between residues D567 and R571. While the LS domain was shown not to be absolutely required for the aminoacylation or editing reactions of LeuRS per $\mathrm{se}^{37}$, it has been shown to be important for tRNA binding and is able to distinguish between the isoacceptors of tRNA ${ }^{\mathrm{Leu}}$ in $E$. coli $^{38}$. Our thermodynamic studies showed a $\sim 3.5$-fold reduction in tRNA ${ }^{\text {Leu }}$ affinity between LeuRS ${ }_{\mathrm{At}}$ and AgnB2 (Supplementary Tables 1 and 2), raising the possibility that the modification of tRNA interacting elements in AgnB2 may play a role in TM84 resistance (tested below).

It has been reported that residues distant from the active site can also be crucial in forming interactions with the negatively charged backbone of the tRNA ${ }^{\text {Leu }}$ CCA $3^{\prime}$-end and its repositioning in catalysi ${ }^{38}$. Mutation of the residue R418 to a neutral amino acid, such as glutamine, decreased the aminoacylation activity in LeuRS $\mathrm{Ec}_{\mathrm{Ec}}$ by reduction of the residence of the $3^{\prime}$-end in the enzyme active site ${ }^{37,38}$. Importantly, the
AgnB2 structure shows the equivalent residue to R418 in LeuRS $\mathrm{Ec}_{\mathrm{Ec}}$ is the neutrally charged Q413 (Fig. 4). Thus, it is possible that Q413 in AgnB2 has a negative effect on binding of the tRNA-dependent inhibitor TM84.

Identification of permissive mutations in the catalytic core. Having identified possible TM84 sensitivity/resistance determinants by comparison of the AgnB2 structure with other LeuRSs known to be sensitive to the toxin, we made mutants of AgnB2 and also LeuRS ${ }_{\text {At }}$ to test the contribution of these sites to antibiotic resistance. Because of the limited number of possible mutants and permutations that could be constructed using this approach, not all potential TM84 resistance/sensitivity determinants could be tested. We initially assayed candidate mutants using a biological assay (see Methods), followed by in vitro enzyme inhibition experiments combined with assessment of direct binding by calorimetry on promising constructs.

Three of the putative resistance determinants in AgnB2, Q573, A102 and T42 described above (Fig. 5a), reside in the catalytic domain and differ from the highly conserved residues in TM84sensitive LeuRSs. We introduced these mutations at corresponding positions (as determined by homology modelling) in the TM84-sensitive enzyme LeuRS ${ }_{\mathrm{At}}(\mathrm{P} 45 \mathrm{~T}, \mathrm{~N} 105 \mathrm{~A}$ and M633Q) and tested whether these mutations imparted resistance using our in vivo assay (Fig. 6a). The single M633Q mutation in the $\mathrm{K}(\mathrm{M} /$ Q)SKS loop is restrictive in nature, as it was not tolerated in the LeuRS $_{\mathrm{At}}$ enzyme, most likely resulting in a functionally inactive enzyme. In contrast, the P45T (Fig. 6a) or N105A (data not
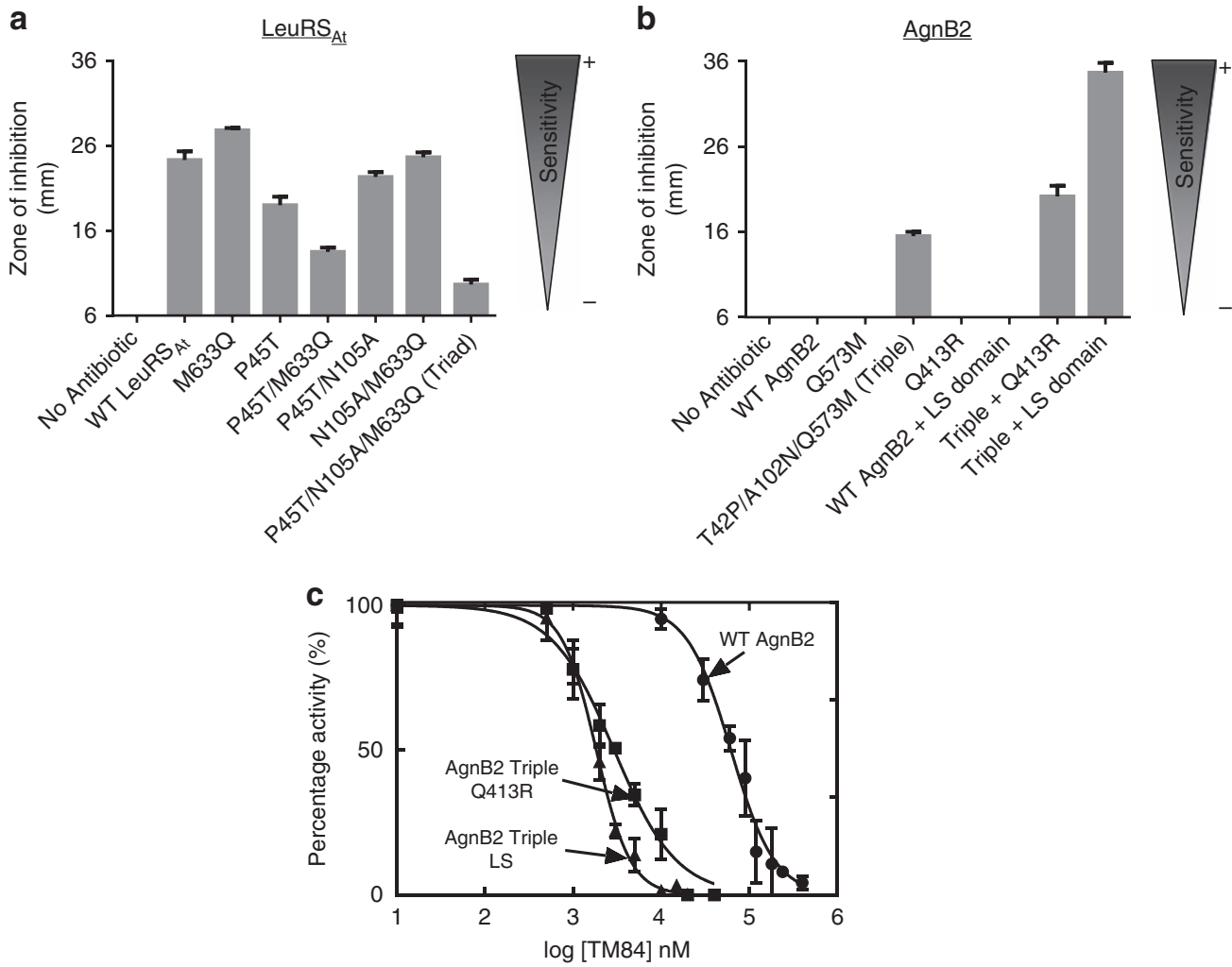

Figure 6 | Comparison of antibiotic sensitivity of wt and mutant forms of LeuRS $\mathbf{A t}_{\mathbf{A t}}$ and AgnB2. A. tumefaciens NTL4 T7pol-Gm-FRT (pYW15d-acs $\triangle a c c R$ ) strain transformed with pBBR1MCS-3 plasmids containing genes encoding wt or mutant LeuRS ${ }_{A t}$ or AgnB2 constructs were tested for their sensitivity towards $50 \mu \mathrm{l}$ of agrocin $84(60 \mu \mathrm{M})$ placed in central well of a bioassay plate. For negative control, sterile ddH $\mathrm{H}_{2} \mathrm{O}$ was used instead of the antibiotic. (a) Bioassay results in graphical form indicating the sensitivity of wt LeuRS $A$ t and LeuRS $\mathrm{At}_{\mathrm{t}}$ mutants; and (b) the resistance properties of wt AgnB2 and selected AgnB2 mutants; (c) Comparison of the TM84 inhibition of the aminoacylation reactions of wt AgnB2 (-), AgnB2 Triple Q413R mutant ( $\mathbf{a})$ and AgnB2 Triple LS domain mutant $(\boldsymbol{\Delta})$. Dose-response curves and the resulting $\mathrm{IC}_{50}$ values were obtained at saturating leucine and (0.675 mM) ATP concentrations (see Methods). Error bars represent s.d. from three independent experiments. 
shown) are permissive mutations and had a small effect on enzyme function or TM84 sensitivity. A P45T/N105A double mutant showed no increase in resistance for LeuRS $\mathrm{At}_{\mathrm{A}}$. When the $\mathrm{P} 45 \mathrm{~T}$ was introduced with M633Q in a double mutant, the P45T acted as a permissive mutation, increasing the resistance of the mutant enzyme. Finally, when all three mutations were introduced into LeuRS ${ }_{\text {At }}$ (P45T/N105A/M633Q, termed LeuRS Triad) there was a significant increase in resistance to the antibiotic (Fig. 6a) compared with wild type (wt).

Kinetic inhibition experiments on LeuRS $S_{\text {At }}$ Triad showed the enzyme was resistant to to concentrations of TM84 up to $500 \mathrm{nM}$ (Supplementary Fig. 6a). This is in contrast to wt LeuRS ${ }_{\mathrm{At}}$, which is sensitive to very low concentrations of TM84 $\left(K_{\mathrm{i}}\right.$ app $=0.3 \pm 0.1$ $\mathrm{nM})^{23}$. The introduction of the Triad mutations also negatively affected the percentage of active enzyme, presumably because these modified residues not only alter engagement of the enzyme with the antibiotic but also modify the interaction of the active site with its substrates. Subsequent thermodynamic analysis of the binding of TM84 to the LeuRS ${ }_{\text {At }}$ Triad alone and the LeuRS Triad pre-incubated with $\mathrm{tRNA}^{\mathrm{Leu}}$ showed respective 10 -fold and 28 -fold decreases in binding affinity, as compared with LeuRS At $_{\text {t }}$ (Supplementary Table 2).

To see if the three reverse mutations in AgnB2 could make the enzyme more sensitive to TM84, a variant containing all three mutations (T42P/A102N/Q573M termed AgnB2 Triple) was constructed. The AgnB2 Triple showed a distinct increase in TM84 sensitivity (larger clearance zone) as detected by bioassay (Fig. 6b). An individual, Q573M mutant did not increase TM84 sensitivity of AgnB2 (Fig. 6b). Kinetic analysis of the AgnB2 Triple mutant showed a 1.8-fold increase in resistance using a fixed concentration of ligand (Supplementary Fig. 6b). Thermodynamic analysis of the binding of TM84 to the AgnB2 Triple alone showed no significant effect on TM84 binding, while there was a 3.5-fold increase in binding of TM84 to the AgnB2•tRNA ${ }^{\text {Leu }}$ complex (Supplementary Table 1).

We also tested whether disruption of the H-bond interaction between Q573 of the KQSKS loop and T581 side chain (Fig. 5a) could affect TM84 binding by mutating the threonine residue to a valine. This single $\mathrm{T} 581 \mathrm{~V}$ mutation, as well as the double mutation (Q573M/T581V) had no detectable effect on TM84 resistance in our bioassays (Supplementary Fig. 7a). However, addition of the T581V mutation to the AgnB2 Triple construct did produce an apparent increase in antibiotic sensitivity (Supplementary Fig. 7a).

\begin{abstract}
A miniloop substitutes for the LS domain in AgnB2 with implications for TM84-resistance and aminoacylation. Since AgnB2 lacks the LS domain and possesses a short peptide with a unique salt bridge between residues D567 and R571 (Fig. 5a), we tested if removal of the salt bridge had any effect on TM84 sensitivity. The salt bridge was disrupted using a number of mutations (D567N, R571E, M and Q), and tested using the bioassays. No significant change in resistance was detected for these mutants compared with wt AgnB2 (Supplementary Fig. 7b). An L577K mutation designed to compete with R571 (Fig. 5) for the salt bridge to the residue D567 produced a clearing zone in our bioassays (Supplementary Fig. 7b) but that is likely due to loss of stability of the enzyme. Taken together, these results indicate that disruption of the D567-R571 salt bridge that replaces the LS domain is not sufficient to cause resistance to TM84, but it may play still play an important structural or canonical catalytic role in AgnB2.
\end{abstract}

Conversion of a TM84-resistant enzyme into a sensitive form by altering key tRNA interactions. To test whether an LS domain can have a substantial effect on the TM84 sensitivity of a LeuRS, we examined recombinant LeuRS H. pylori (LeuRS Hp $_{\text {) }}$, which lacks an LS domain (Fig. 4). Interestingly, we found using in vitro aminoacylation assays that the LeuRS $_{\mathrm{Hp}}$ was still significantly inhibited by TM84 $\quad\left(\mathrm{IC}_{50}=34 \pm 1.14 \mathrm{nM}\right.$; Supplementary Fig. 8) although to a lesser degree than LeuRS (ref. 23). Interestingly, this enzyme also contains a neutral Q413 residue in a homologous position to $\mathrm{R} 418$ from LeuRS $\mathrm{EC}$ is thought to be involved in positioning of the tRNA CCA $3^{\prime}$-end in the enzyme active site ${ }^{25,38}$. We took this one step further, by introducing all three core mutations implicated in TM84 resistance in AgnB2 into LeuRS ${ }_{\mathrm{Hp}}$ (P40T, N100A and M573Q). However, our in vitro aminoacylation analysis revealed that this enzyme was inactive under the conditions used in our study (Supplementary Fig. 8).

To pursue further a possible role of the LS domain in TM84 sensitivity, we inserted the LS domain from LeuRS ${ }_{\mathrm{At}}$ into AgnB2 LeuRS and tested the chimeric enzyme's sensitivity to TM84 in our bioassay. In addition, we also inserted the LS domain into the background of the AgnB2 Triple variant (T42P/A102N/Q573M). Our results show that insertion of the LS domain into AgnB2 wt had no effect on antibiotic sensitivity in our bioassays but when the LS domain was inserted into the AgnB2 triple background there was a substantial increase in clearing zone size (Fig. 6b). A comparison of the TM84 inhibition of aminoacylation activity of the AgnB2 Triple mutant + LS domain construct against wt AgnB2, at a fixed ATP concentration of $0.675 \mathrm{mM}$ ATP (where no substrate inhibition of either enzyme is detected) showed a 34 -fold $\left(\mathrm{IC}_{50}\right.$ of $\left.1.8 \mu \mathrm{M}\right)$ decrease in the $\mathrm{IC}_{50}$ of the chimeric mutant compared with wild-type ( $\mathrm{IC}_{50}$ of $61.3 \mu \mathrm{M}$ ) (Fig. $6 \mathrm{c}$ ). ITC analysis of this construct was used to deconvolute the contribution of tRNA to the binding of TM84 to the mutant. Our results showed that TM84 binding alone $\left(K_{\mathrm{d}}=258 \mathrm{nM}\right)$ increased by fourfold (Fig. 7a) and TM84 binding in presence of tRNA $\left(K_{\mathrm{d}}=19 \mathrm{nM}\right)$ increased by 17 -fold respectively, compared with wt AgnB2 (Fig. 7b).

The positively charged $\mathrm{R} 418$ residue in $\mathrm{LeuRS}_{\mathrm{Ec}}$ has been implicated in controlling the translocation of the negatively charged backbone of the tRNA ${ }^{\text {Leu }}$ CCA $3^{\prime}$-end into the synthetic active site ${ }^{25,38}$. We tested whether modification of the homologous position in AgnB2, Q413, from a neutral to positively charged arginine might modulate TM84 resistance through stabilization of the tRNA-dependent inhibition complex. Introduction of a single-point Q413R mutation into AgnB2 showed no effect on the size of clearing zones in our bioassays compared with wt (Fig. 6b). However, when we placed the Q413R mutation in the background of the AgnB2 Triple mutant (T42P/ A102N/Q573M) there was a substantial increase in the clearing zone diameter suggesting an increase in TM84 sensitivity compared with the AgnB2 Triple (Fig. 6b). Analysis of the TM84 inhibition kinetics of the AgnB2 Triple + Q413R mutant showed an $\mathrm{IC}_{50}$ of $2.9 \mu \mathrm{M}, 21$-fold smaller than wt-AgnB2 at a fixed ATP concentration (Fig. 6c) indicating this construct had a significant increase in TM84 sensitivity compared with wt. We then analyzed the thermodynamics of binding of TM84 to the AgnB2 Triple Q413R•tRNA ${ }^{\text {Leu }}$ complex and found it was sixfold tighter than wt AgnB2 (Fig. 7b). However, the binding of TM84 to the AgnB2 Triple Q413R enzyme alone was only 1.3-fold tighter than the wt enzyme (Fig. 7a). This further suggested the importance of tRNA interacting residues for imparting sensitivity towards TM84.

In summary, our results suggest that the absence, or modification of, idiosyncratic tRNA binding elements in AgnB2, act cumulatively with critical core mutations on the periphery of the LeuRS active site, significantly contributing to the high degree of TM84 resistance observed in AgnB2. 
a

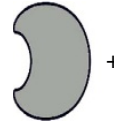

AgnB2

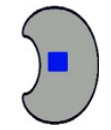

AgnB2•TM84

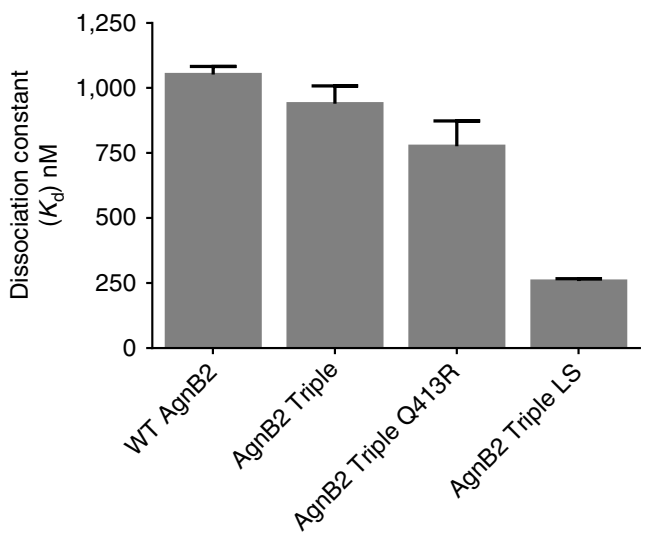

b
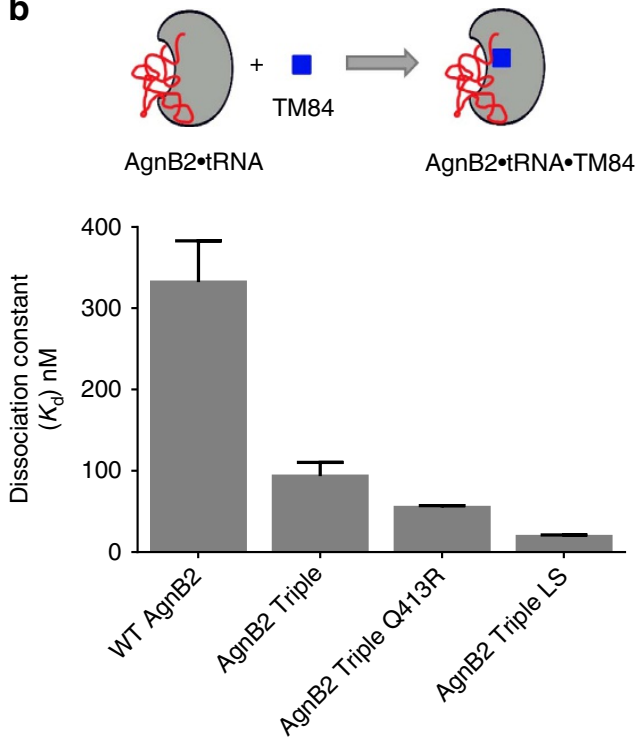

Figure 7 | The accumulative mutation of tRNA interacting elements with core Triple mutations in AgnB2 increases the enzyme's affinity to TM84. ITC results depicted in graphical form compare the TM84-binding affinities of wt AgnB2 and Triple AgnB2 mutant \pm Q413R or LS domain to (a) TM84 alone; or (b) TM84 in the presence of tRNA ${ }^{\text {Leu }}$. Error bars represent standard deviation from three independent experiments.

\section{Discussion}

TM84 (agrocin 84) is a highly potent LeuRS toxin that employs a unique tRNA-dependent inhibition mechanism. During production of agrocin 84 , the biocontrol $A$. radiobacter strain 84 expresses the self-immunity LeuRS AgnB2 to prevent cell suicide. Our data show that AgnB2 achieves resistance to TM84 by minimizing the effect tRNA ${ }^{\text {Leu }}$ has on the toxin's affinity for the enzyme as well as weakening its affinity for tRNA ${ }^{\text {Leu }}$ and the inhibitor alone. Remarkably, this resistance phenotype is achieved without dramatically affecting the catalysis of the overall aminoacylation reaction (Table 1). Consistent with this observation, the X-ray crystal structure of the AgnB2•tRNA ${ }^{\text {Leu }} \bullet$ Leu-AMS ternary complex reveals that residues in the synthetic active site that directly bind Leu-AMP are not substantially different from other bacterial LeuRSs.

We identified key molecular determinants imparting TM84 resistance (or sensitivity) to LeuRSs by utilizing an approach based on the analysis of structures of the naturally resistant and sensitive enzymes AgnB2 and LeuRS $\mathrm{Ec}_{\mathrm{Ec}}$ (Fig. 8). LeuRS mutants that affected TM84 sensitivity were then tested using in vitro and in vivo approaches. Our data indicate that the molecular elements in LeuRSs that contribute to TM84 sensitivity or resistance are much more complex than one would expect a priori. Surprisingly, some of these determinants were not located directly in the active site but on the periphery (with distances $\sim 10-15 \AA$ for most residues and $25 \AA$ for Q413), including some residues interacting with the tRNA ${ }^{\mathrm{Leu}}$. The core resistance mutations in the catalytic domain of TM84 resistant AgnB2 are located in KQSKS loop (Q573), just upstream of the HIGH loop (T42) or buttressing next to T42 in the case of A102. It is possible that the nature of the amino acids at these particular sites in LeuRSs may affect the molecular dynamics of these two critical catalytic motifs and ultimately altering the LeuRSs TM84 sensitivity phenotype. The exact contribution of other determinants to TM84 sensitivity at a greater distance from the active site is more difficult to discern. For AgnB2, alterations in tRNA-binding contacts, due to lack of an LS domain, C-term domain modifications, and the effect of Q413 on CCA $3^{\prime}$-end localization in the active site, may all play a role in destabilizing a TM84 ternary complex leading to resistance. It maybe that evolution has traversed a narrow energetic landscape towards a resistance phenotype by exploiting small energetic differences between mutants to produce larger scale changes in only certain kinetic and/or thermodynamic parameters. Presumably, there must be other additional unconserved residues that have played subtle compensatory roles on the pathway towards evolution of TM84 resistance.

Why did nature evolve TM84 to target LeuRS At $_{\text {and not }}$ another AARSs from A. tumefaciens, and in particular the other Class I AARSs that have similar active site architectures such as IleRS and ValRS. TM84 does appear to be LeuRS specific, as the antibiotic does not inhibit agrobacterial IleRS ${ }^{12}$, and the single LeuRS agnb2 gene is capable of providing agrocin 84-immunity to the pathogen. In silico docking analysis of TM84, from the LeuRS $_{\mathrm{Ec}}$ structure (PDB: 3ZGZ), into the catalytic domain of existing IleRS (PDB code: 1JZQ) and ValRS (PDB code: 1GAX) structures reveals steric clashes of the 'leucine-like' region of TM84 with hydrophobic side chains in the active site (Supplementary Fig. 9). This analysis may provide some understanding of the LeuRS specificity of TM84. One possible reason that agrocin $84 / \mathrm{TM} 84$ evolved to target a LeuRS maybe because leucine is one of the most abundant amino acids in proteins, and so translation could be particularly sensitive to interference of LeuRS activity. A more plausible reason is that agrocin 84/TM84, or its evolutionary ancestor, had other roles in Agrobacterium biology that predisposed the toxin to evolve into a LeuRS inhibitor. The recent finding that the uptake molecule of agrocin 84, when cleaved from TM84, can act as signalling molecule binding to a key agrobacterial transcriptional regulator that controls quorum-sensing signal synthesis ${ }^{9}$ provides some support for this latter view.

Horizontal gene transfer of the agnB2 gene on the pAgK84 plasmid from $A$. radiobacter strain K84 to the pathogen threatens emasculation of the biocontrol ${ }^{5,16,17}$. Genetic engineering of pAgK84 to prevent conjugative transfer of the plasmid to the pathogen has resulted in A. radiobacter K1026 strain ${ }^{39}$, the first bioengineered microbe approved for release into the environment ${ }^{40}$. Yet despite not containing any foreign DNA, this genetically altered strain is still only registered for use as a biopesticide in a limited number of countries (Gary Bullard and Allen Kerr, personal communications). In addition, as with all 

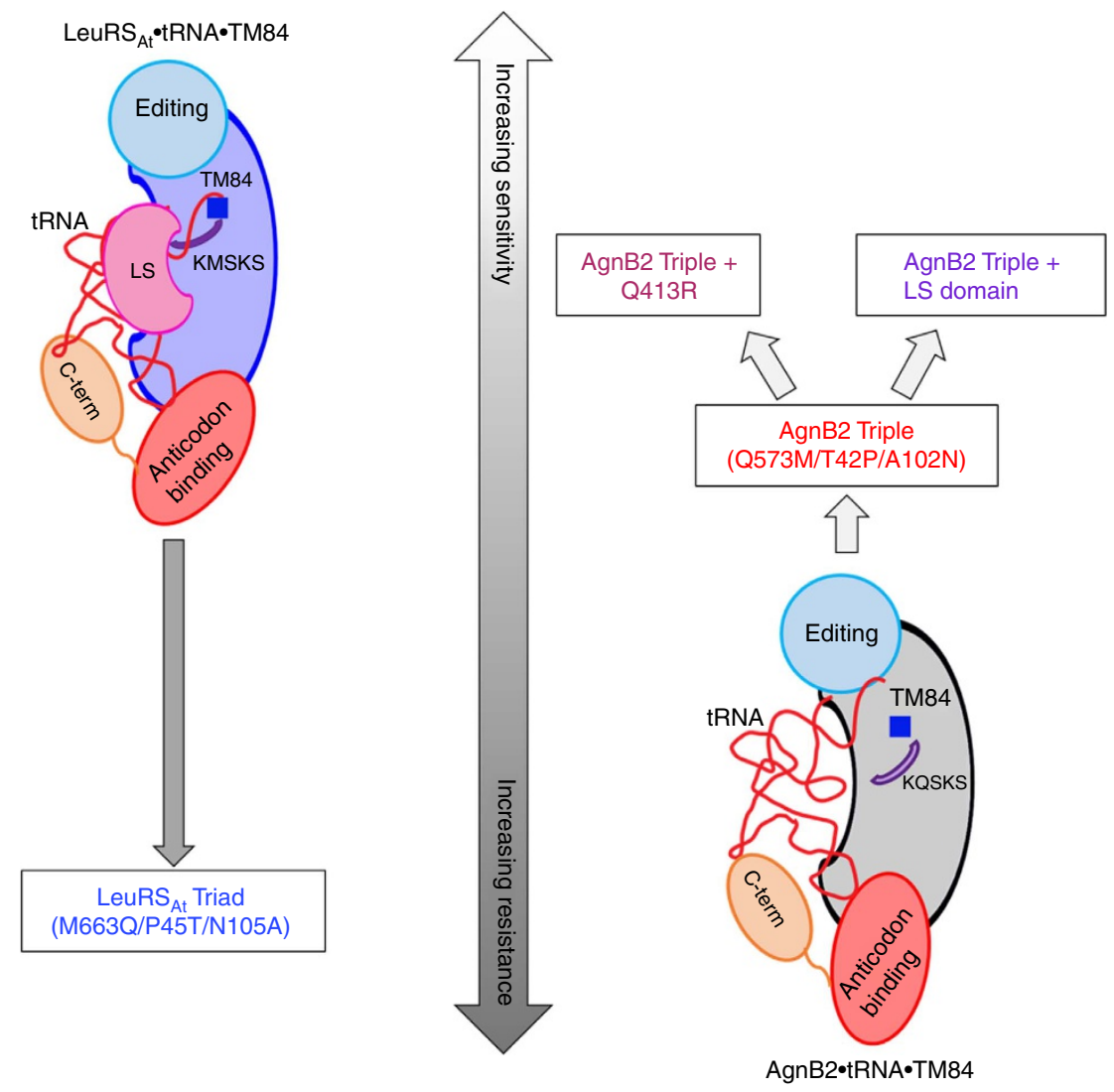

Figure 8 | Model illustrating determinants of TM84 sensitivity and resistance in LeuRS $\mathbf{A t}_{\mathbf{A t}}$ and $\mathbf{A g n B 2}$. The ternary complex of LeuRS $\mathrm{At}_{\mathrm{t}}$ forms a tight binding complex with the CCA 3 '-end of the tRNA and TM84. Upon mutation of three critical residues, P45, M633 and N105, a TM84 resistant mutant (LeuRS $_{A t}$ Triad) is obtained. AgnB2 on the other hand forms a weak ternary complex with tRNA and TM84, thereby explaining its high level of resistance

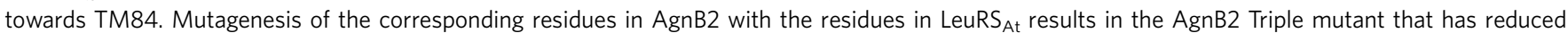
levels of AgnB2 resistance. Upon introduction of Q413R mutation or addition of a LS-domain to the AgnB2 triple mutant, increased sensitivity towards TM84 is achieved. Thus these residues along with the LS domain may be the key determinants of sensitivity or resistance in LeuRS $\mathrm{At}_{\mathrm{a}}$ and $\mathrm{AgnB}_{\mathrm{B}}$.

antibiotic interventions, continued success against resistance can never be completely assured. Thus, understanding the resistance mechanism of agrocin 84 immunity protein AgnB2 not only provides key insights into the mechanism of a fascinating tRNA-dependent toxin but also informs research into the continued crop protection provided by this biocontrol against the spectre of antibiotic resistance.

\section{Methods}

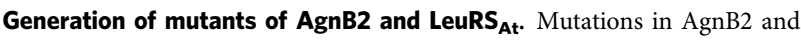
LeuRS $_{\text {At }}$ were introduced using the Quikchange site-directed mutagenesis method (Stratagene). Mutagenesis was carried out in the agnB2 gene previously cloned ${ }^{12}$ into the pET-28a vector (Novagen) for use in recombinant protein expression, and also in the agnB2 gene subcloned into the broad host range plasmid pBBR1MCS-3

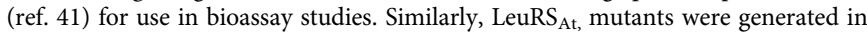
the A. tumefaciens leuS gene cloned into the pET-21b vector (for recombinant protein expression) and pBBR1MCS-3 (bioassays). Multiple rounds of mutagenesis were performed to obtain more than one mutation in an enzyme. DNA sequence analysis was used to confirm the correct construction of the entire gene sequence for all single, and multiple site mutants, used in this study.

Insertion of the LS domain of LeuRS At $_{\mathbf{t}}$ into AgnB2. The LS domain of LeuRS (141 bp) was inserted into AgnB2 (replacing the region between amino acid no. 566 and 572 with the LS domain) ${ }^{37}$ To obtain this, the forward PCR primer was designed to contain the region of AgnB2 ( $\sim 25 \mathrm{bp}$ ) preceding the LS domain insertion followed by the first 25 bases of the LS domain $\left(5^{\prime}-3^{\prime}\right)$. Similarly, the reverse primer was designed so as to include the last $25 \mathrm{bp}$ of the LS domain $\left(3^{\prime}-5^{\prime}\right)$ and the gene sequence of AgnB2 after the LS domain insertion. An extension reaction was carried out to obtain mega primers, (forward and reverse) encoding the entire LS domain (of LeuRS ${ }_{\mathrm{At}}$ ) flanked by the sequence of AgnB2 on both sides using the protocol by Wang and Malcolm ${ }^{42}$. DpnI digestion was then carried out to remove the wt template. The resultant mega primer was gel purified using the
Qiaex II gel extraction kit (Qiagen) to be used in the second step. The PCR amplified mega primers (generated in the first step) were then added to the extension reaction in presence of the pET-28a plasmid containing the agnB2 gene (using the Quikchange site-directed mutagenesis protocol II). Positive clones encoding AgnB2 with the entire LS domain of LeuRS PCR and finally confirmed by DNA sequencing analysis. Using a similar strategy, LS domain was added to the AgnB2 Triple mutant as well as the AgnB2 Triple Q413R mutant.

Construction of A. tumefaciens bioassay strains. A modified acs operon encoding the agrocin 84 transporter system (agrocinopine permease) ${ }^{43-45}$ was cloned into pYW15d plasmid containing a T5 promoter and an antibiotic marker (carbenicillin) ${ }^{46}$. Importantly, the $\operatorname{acc} R$ repressor gene, responsible for the regulation of the agrocinopine permease ${ }^{47}$, had been removed from our construction in order to produce A.tumefaciens strains with maximum agrocin 84 uptake. The pYW15d-acs $\triangle a c c R$ plasmid encoding the agrocin 84 transporter and the pBBR1MCS-3 plasmid $^{41}$ containing the agnB2/leu $S_{A t}$ gene and a T7 promoter and a tetracycline marker were then transformed into an electrocompetent A. tumefaciens NTL4 T7pol-Gm-FRT strain containing the DE3 lysogen encoding a T7 RNA polymerase ${ }^{48}$. Briefly, electroporation was performed at $2400 \mathrm{~V}$ and $400 \Omega$ and the culture was then grown in $3 \mathrm{ml} \mathrm{LB}$ broth for $2.5 \mathrm{~h}$. The A. tumefaciens culture was then streaked on LB agar plates containing $100 \mu \mathrm{g} \mathrm{ml}^{-1}$ of carbenicillin, $15 \mu \mathrm{g} / \mathrm{ml}$ of gentamycin and $10 \mu \mathrm{g} / \mathrm{ml}$ of tetracycline. The final construct $A$. tumefaciens NTL4 T7pol-Gm-FRT containing both pYW15d-acs $\triangle a c c R$ (encoding the agrocin 84 transporter) and a pBBR1MCS-3 plasmid (containing a gene encoding either wt or mutant AgnB2/LeuRS ${ }_{\text {At }}$ proteins was then used for bioassay analysis. Individual colonies were picked and grown in $3 \mathrm{ml}$ cultures to make glycerol stocks for storage at $-80^{\circ} \mathrm{C}$.

Protein expression and purification. All enzymes (mutants of AgnB2 and LeuRS $_{\text {At }}$ ) were overexpressed in E. coli BL21 (DE3) RIL codon plus cells (Agilent) (containing pET-28a plasmid encoding C-terminal 6X His-tagged AgnB2 gene or pET-21b plasmid encoding C-terminal 6X histidine tagged LeuRS At $_{\text {gene). }}$ gen 
LeuRS enzymes were purified in a similar manner as detailed previously for LeuRS $_{\mathrm{At}}{ }^{23}$. In brief, His-tagged proteins were purified by affinity chromatography using Ni-NTA agarose beads (Qiagen), followed by purification using a strong anion-exchange Mono-Q column (GE Healthcare) by FPLC. All proteins were confirmed to be $\sim 95 \%$ pure using SDS-polyacrylamide gel electrophoresis analysis and the active concentration determined by active-site titration.

Purification of TM84. Agrocin 84 and TM84 were obtained from A. tumefaciens NT1 (pAgK84::Tn5A1-B5) agrocin 84 secretion strain grown at $28^{\circ} \mathrm{C}$ in D-glucose minimal media supplemented with $50 \mu \mathrm{g} \mathrm{ml}^{-1}$ kanamycin as described previously ${ }^{9,23}$. Briefly, agrocin 84 and TM 84 was separated from other media components by adsorption on charcoal, and then eluted using $70-90 \%$ reagent alcohol, dried and both further purified by reverse-phase HPLC. The concentration of TM84 was determined using an extinction coefficient $(\varepsilon=260 \mathrm{~nm})$ of $0.0154 \mathrm{M}^{-1} \mathrm{~cm}^{-1}$.

Bioassays. In vivo assays to determine the agrocin 84 sensitivity of AgnB2/ LeuRS $_{\mathrm{At}}$ and their mutants were performed. Stationary phase cultures of $A$. tumefaciens NTL4 T7pol-Gm-FRT strains transformed with both pYW15d-acs $\triangle a c c R$ (encoding the agrocin 84 transporter) and the pBBR1MCS-3 plasmids (containing genes encoding the wt or mutant AgnB2/LeuRS $\mathrm{At}_{\mathrm{At}}$ proteins) were first diluted 1:100 and then added to $20 \mathrm{ml} \mathrm{LB}$ agar plates containing $100 \mu \mathrm{g} / \mathrm{ml}$ of carbenicillin, $15 \mu \mathrm{g} \mathrm{ml}^{-1}$ of gentamycin, $10 \mu \mathrm{g} \mathrm{ml}^{-1}$ of tetracycline and $1 \mathrm{mM}$ IPTG. A well was punched in the centre of the plate and $50 \mu \mathrm{l}$ of $60 \mu \mathrm{M}$ agrocin 84 was added to determine the sensitivity of agrocin 84 towards AgnB2 and LeuRS $A$. The plates were then incubated at $28^{\circ} \mathrm{C}$ for $48 \mathrm{~h}$. The sensitivity of the strain towards agrocin 84 was determined by measuring the zone of clearance around the well containing agrocin 84 . The control strain containing only the acs operon, but no pBBR1MCS-3 plasmid showed a zone of clearance of around $4 \mathrm{~cm}$. Therefore, any sample having a zone of clearance $>4.1 \mathrm{~cm}$ was considered inactive.

tRNA ${ }^{\text {Leu }}$ transcription. In this study, we used purified in vitro transcribed tRNA ${ }^{\mathrm{Leu}}(\mathrm{UAA})$ isoacceptor based on the nucleotide sequence from $A$. tumefaciens (referred to in text as agrobacterium tRNA ${ }^{\mathrm{Leu}}$ ) and not $A$. radiobacter. This allowed a direct comparison of in vitro aminoacylation kinetics and ITC analysis with our in vivo studies of AgnB2 mutants. Importantly, both tRNA ${ }^{\text {Leu }}$ isoacceptors from both species share a substantial similarity. Agrobacterium tRNA ${ }^{\text {Leu }}$ with (UAA) isoacceptor was obtained by treating plasmids encoding the tRNA ${ }^{\mathrm{Leu}}$ gene with $B s t \mathrm{NI}$ overnight at $60^{\circ} \mathrm{C}$. The linearized DNA was then used as a template in the in vitro transcription reaction containing $40 \mathrm{mM}$ Tris $\mathrm{HCl}(\mathrm{pH} 8.0), 25 \mathrm{mM} \mathrm{MgCl}_{2}$, $40 \mathrm{mM}$ DTT, $0.1 \%$ Triton X-100, $1 \mathrm{mM}$ spermidine and $2 \mathrm{mM} \mathrm{rNTPs}, 1 \mathrm{Uml}^{-1}$ RNase inhibitor and $9 \mu \mathrm{M}$ T7 RNA polymerase ${ }^{23}$. After DNase I treatment for removal of template DNA and quenching the reaction with EDTA, the tRNA was precipitated using $0.3 \mathrm{M}$ sodium acetate and absolute ethanol. The tRNA obtained was further purified on a 12\% denaturing PAGE gel (19:1) containing $8 \mathrm{M}$ urea and 1X TBE buffer ( $\mathrm{pH}$ 8.3). Gel extraction of the tRNA was then performed, followed by refolding of tRNA at room temperature using $1 \mathrm{mM} \mathrm{MgCl}$ after incubating $1 \mathrm{~min}$ at $80^{\circ} \mathrm{C}$ (refs 23,49$)$. The concentration of tRNA was determined and corrected by a factor of 1.34 to account for the hypochromic effect on absorbance ${ }^{23}$

Active-site titration assay. Active enzyme concentrations of AgnB2, LeuRS ${ }_{A t}$ and their respective mutants was determined using the method described by Beebe et al. ${ }^{50}$ and Ferst ${ }^{51}$. In brief, purified enzymes in the range of $1-2 \mu \mathrm{M}$ (as determined by the Bio-Rad Protein assay) were added to a reaction mix containing $20 \mathrm{mM} \mathrm{KCl}, 10 \mathrm{mM} \mathrm{MgCl} 2,10 \mathrm{mM} \beta$-mercaptoethanol ( $\beta$-ME), $1 \mathrm{mM}$ L-leucine, $5 \mu \mathrm{g} \mathrm{ml}^{-1}$ yeast inorganic pyrophophatase (NEB), $5 \mu \mathrm{M}$ ATP and $4 \mu \mathrm{Ci}[\gamma-32 \mathrm{P}]$ ATP. The reaction was initiated at $28^{\circ} \mathrm{C}$ and $5 \mu \mathrm{l}$ aliquots of the reaction quenched using 7\% perchloric acid and $1 \mathrm{M} \mathrm{HCl}$ over a time period of 5-30 min. The amplitude of the burst phase of Leu-AMP formation was used to determine the amount of Leu-AMP formed and subsequently the active concentration of the enzymes.

ATP/PPi exchange assay. The formation of Leu-AMP by ATP/PPi exchange assays was performed as described earlier ${ }^{23,50}$. Briefly, the reaction buffer contained $50 \mathrm{mM}$ HEPES buffer (pH 7.4), $20 \mathrm{mM} \mathrm{KCl}, 10 \mathrm{mM} \mathrm{MgCl} 2,500 \mu \mathrm{M}$ L-leucine, $4 \mathrm{mM}$ ATP, $1 \mathrm{mM}$ tetrasodium pyrophosphate $(\mathrm{NaPPi}), 10 \mu \mathrm{Ci} / \mathrm{mmol}\left[{ }^{32} \mathrm{P}\right] \mathrm{NaPPi}$ and $1 \mu \mathrm{M}$ bovine serum albumin (BSA). The reaction was initiated by addition of $1-4 \mathrm{nM}$ of active enzyme and incubated at $28^{\circ} \mathrm{C}$. Aliquots (5 $\mu \mathrm{l}$ each) were collected over $30 \mathrm{~min}$ and quenched in a $10 \%$ charcoal slurry containing $0.5 \% \mathrm{HCl}$ and $50 \mu \mathrm{l}$ of $200 \mathrm{mM}$ sodium pyrophosphate in $1 \mathrm{M} \mathrm{HCl}$. Liquid scintillation counting was then used to measure the amount of $\left[{ }^{32} \mathrm{P}\right]-\mathrm{PPi}$ incorporation into ATP that was adsorbed onto the charcoal.

Aminoacylation assay. Aminoacylation activities of AgnB2, LeuRS ${ }_{A t}$ and their mutants were measured in $50 \mathrm{mM}$ HEPES buffer $(\mathrm{pH} 7.4)$ containing $20 \mathrm{mM} \mathrm{KCl}$, $25 \mathrm{mM} \mathrm{MgCl}_{2}, 25 \mathrm{mM} \beta$-ME, $4 \mathrm{mM}$ ATP, $500 \mu \mathrm{M}\left[3,4,5{ }^{3} \mathrm{H}\right]$ L-leucine
( $20 \mu \mathrm{Ci} / \mathrm{mmole}$ ), $5 \mu \mathrm{g} / \mathrm{ml}$ inorganic pyrophosphatase, $1 \mu \mathrm{M}$ BSA, $10 \mu \mathrm{M}$ of active in vitro transcribed tRNA ${ }^{\mathrm{Leu}}$ and enzymes in the range of $2-8 \mathrm{nM}$. Reactions were pre-incubated with or without substrate and/or inhibitor and then carried out at $28^{\circ} \mathrm{C}\left(37^{\circ} \mathrm{C}\right.$-LeuRS $\left.\mathrm{Hp}_{\mathrm{p}}\right)$. Aliquots of the reaction $(5-10 \mu \mathrm{l})$ were quenched on $3 \mathrm{mM}$ filter pads (Whatman, GE Healthcare) presoaked with $5 \%$ trichloroacetic acid (TCA). The pads were washed thrice with $5 \%$ TCA and $90 \%$ ethanol at $4{ }^{\circ} \mathrm{C}$ and then dried. The amount of Leu-tRNA ${ }^{\mathrm{Leu}}$ was then quantified using scintillation counting. Each reaction was performed in triplicate and analyzed using Kaleidagraph (for $K_{\mathrm{m}}$ and $k_{\text {cat }}$ determinations) or GraphPad Prism software (for $\mathrm{IC}_{50}$ determination).

Isothermal titration calorimetry. The affinity of TM84 binding to AgnB2, LeuRS $_{\mathrm{At}}$ and their mutants was determined using ITC as described earlier ${ }^{23}$. Binary interactions (TM84 binding to protein) and ternary interactions (TM84 binding to protein + tRNA (1:1.2 molar ratio)) were tested using an Auto-ITC200 microcalorimeter (from MicroCal/GE Healthcare). Typically, about $4-8 \mu \mathrm{M}$ active protein solution and a 10 -fold higher ligand concentration were prepared in buffer containing $50 \mathrm{mM}$ HEPES (pH 7.4), $20 \mathrm{mM} \mathrm{KCl} 10 \mathrm{mM} \mathrm{MgCl}_{2}$ and $1 \mathrm{mM} \beta$-ME The titrations were carried out in triplicate at $28^{\circ} \mathrm{C}$. The data was then analyzed using Origin software (version 7) and fitted to a one-site or a two-site binding model to obtain the binding affinity $\left(K_{\mathrm{d}}\right)$, stoichiometry $(\mathrm{N})$ and enthalpy $(\Delta \mathrm{H})$ for each complex.

Crystallization. Crystallization was carried out at $20^{\circ} \mathrm{C}$ by the hanging drop vapor diffusion method. Crystals of the ternary complex AgnB2 LeuRS•tRNA ${ }^{\text {Leu }} \bullet$ LeuAMS were obtained by mixing $1 \mu \mathrm{l}$ of a solution containing AgnB2 LeuRS at $43 \mu \mathrm{M}, \mathrm{tRNA}^{\mathrm{Leu}}$ (A. tumefaciens) at $34 \mu \mathrm{M}$ and Leu-AMS at $2 \mathrm{mM}$; with $1 \mu \mathrm{l}$ of reservoir solution containing $0.1 \mathrm{M} \mathrm{MES}(\mathrm{pH} 6.5), 0.2 \mathrm{M}$ ammonium sulfate and 27\% (w/v) PEG 5000 MME. Quality and size of crystals were optimized by macroseeding with protein at $2 \mathrm{mg} \mathrm{ml}^{-1}$. The crystals were frozen in liquid nitrogen after transfer for a few seconds in the mother liquor plus $15 \%(\mathrm{v} / \mathrm{v})$ ethylene glycol as cryoprotectant.

Structure determination and refinement. The diffraction data sets of the

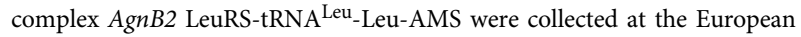
Synchrotron Radiation Facility (ESRF, France). The data sets were integrated and scaled with the XDS suite ${ }^{52}$. Further data analysis was performed with the CCP4 suite $^{53}$. The structure was initially solved by molecular replacement with PHASER $^{54}$ using as a model the catalytic and anticodon-binding domains of a model built with SWISS-Model ${ }^{55}$ based on homology to LeuRS ${ }_{\mathrm{Tt}}$ (PDB 2BTE $)^{56}$ and LeuRS $S_{\mathrm{Ec}}$ (PDB: 4AS1) ${ }^{25}$. The molecular replacement solution was used to search with PHASER for AgnB2 tRNA ${ }^{\text {Leu }}$ using as a model the core of $E$. coli tRNA $^{\text {Leu }}$ (bases 1-73, except the bases belonging to the long-variable arm). The obtained model was completed by manual placing of the ZN1 domain, leucine-specific domain, C-terminal domain and editing domain. Manual adjustments were done with $\mathrm{COOT}^{57}$ and the final model was refined using REFMAC5 (ref. 58). Structure quality was analysed with MOLPROBITY (http://molprobity.biochem.duke.edu/) and showed all residues in allowed regions. Figures were drawn with PYMOL (http://www.pymol.org/).

Molecular modelling. A homology model of LeuRS ${ }_{\mathrm{At}}$ was built using the Insight II Molecular Modeling System (www.accelrys.com) based upon the template structure of the LeuRS ${ }_{\mathrm{Tt}}(\mathrm{PDB} \text { ID } 1 \mathrm{H} 3 \mathrm{~N})^{35}$. A normalized sequence-structure compatibility score of 0.77 was calculated for the LeuRS $\mathrm{At}_{\mathrm{At}}$ homology model using the Verify the three-dimensional module of Insight $\mathrm{II}^{59,60}$.

Data availability. Atomic coordinates and structure factors for the AgnB2 $\bullet$ tRNA $\bullet$ Leu-AMS ternary complex have been deposited in the RCSB Protein Data Bank with the accession code 5AH5. All other data associated with this manuscript are available from the corresponding author on reasonable request.

\section{References}

1. Walsh, C. Antibiotics : Actions, Origins, Resistance 335 (ASM Press, 2003).

2. Wright, G. D. The antibiotic resistome: the nexus of chemical and genetic diversity. Nat. Rev. Microbiol. 5, 175-186 (2007).

3. Levy, S. B. The Antibiotic Paradox: How the Misuse of Antibiotic Destroys Their Curative Powers (Perseus Publishing, 2002).

4. Kerr, A. \& Htay, K. Biological control of Crown Gall through bacteriocin production. Physiol. Plant Pathol. 4, 37-44 (1974).

5. Kerr, A. Biological control of crown gall through production of agrocin 84 Plant Dis. 64, 25-30 (1980).

6. Kerr, A. \& Tate, M. E. Agrocins and the biological control of crown gall. Microbiol. Sci. 1, 1-4 (1984).

7. Roberts, W. P., Tate, M. E. \& Kerr, A. Agrocin 84 is a 6-N-phosphoramidate of an adenine nucleotide analogue. Nature 265, 379-381 (1977)

8. Tate, M. E., Murphy, P. J., Roberts, W. P. \& Kerr, A. Adenine N6-substituent of agrocin 84 determines its bacteriocin-like specificity. Nature 280, 697-699 (1979). 
9. El Sahili, A. et al. A pyranose-2-phosphate motif is responsible for both antibiotic import and quorum-sensing regulation in Agrobacterium tumefaciens. PLoS Pathog. 11, e1005071 (2015).

10. Murphy, P. J., Tate, M. E. \& Kerr, A. Substituents at N6 and C-5' control selective uptake and toxicity of the adenine-nucleotide bacteriocin, agrocin 84 , in Agrobacteria. Eur. J. Biochem. 115, 539-543 (1981).

11. Ryder, M. H., Tate, M. E. \& Jones, G. P. Agrocinopine A, a tumor-inducing plasmid-coded enzyme product, is a phosphodiester of sucrose and L-arabinose. J. Biol. Chem. 259, 9704-9710 (1984).

12. Reader, J. S. et al. Major biocontrol of plant tumors targets tRNA synthetase. Science 309, 1533 (2005).

13. Ryder, M. H., Slota, J. E., Scarim, A. \& Farrand, S. K. Genetic analysis of agrocin 84 production and immunity in Agrobacterium spp. J. Bacteriol. 169, 4184-4189 (1987).

14. Kim, J. G. et al. Bases of biocontrol: sequence predicts synthesis and mode of action of agrocin 84, the Trojan horse antibiotic that controls crown gall. Proc. Natl Acad. Sci. USA 103, 8846-8851 (2006).

15. Farrand, S. K., Slota, J. E., Shim, J. S. \& Kerr, A. Tn5 insertions in the agrocin 84 plasmid: the conjugal nature of pAgK84 and the locations of determinants for transfer and agrocin 84 production. Plasmid 13, 106-117 (1985).

16. Panagopoulos, C. G., Psallidas, P. G. \& Alivizatos, A. S. in Soil-Borne Plant Pathogens. (ed. Schiffers, B.a.G.,W.) 569-578 (Academic Press, 1979).

17. Ellis, J. G. \& Kerr, A. in Soil-Borne Plant Pathogens. (ed. Schiffers, B.a.G.,W.) 579-583 (Academic Press, 1979).

18. Schimmel, P., Tao, J. \& Hill, J. Aminoacyl tRNA synthetases as targets for new anti-infectives. FASEB J. 12, 1599-1609 (1998).

19. Dewan, V., Reader, J. \& Forsyth, K. M. Role of aminoacyl-tRNA synthetases in infectious diseases and targets for therapeutic development. Top. Curr. Chem. 344, 293-329 (2014).

20. Hernandez, V. et al. Discovery of a novel class of boron-based antibacterials with activity against gram-negative bacteria. Antimicrob. Agents Chemother. 57, 1394-1403 (2013).

21. Hughes, J. \& Mellows, G. Inhibition of isoleucyl-transfer ribonucleic acid synthetase in Escherichia coli by pseudomonic acid. Biochem. J. 176, 305-318 (1978).

22. Hughes, J. \& Mellows, G. On the mode of action of pseudomonic acid: inhibition of protein synthesis in Staphylococcus aureus. J. Antibiot. (Tokyo) 31, 330-335 (1978).

23. Chopra, S. et al. Plant tumour biocontrol agent employs a tRNA-dependent mechanism to inhibit leucyl-tRNA synthetase. Nat. Commun. 4, 1417 (2013).

24. Chopra, S. \& Reader, J. tRNAs as antibiotic targets. Int. J. Mol. Sci. 16, 321-349 (2014).

25. Palencia, A. et al. Structural dynamics of the aminoacylation and proofreading functional cycle of bacterial leucyl-tRNA synthetase. Nat. Struct. Mol. Biol. 19, 677-684 (2012).

26. Rock, F. L. et al. An antifungal agent inhibits an aminoacyl-tRNA synthetase by trapping tRNA in the editing site. Science 316, 1759-1761 (2007).

27. Kobayashi, T. et al. Structural snapshots of the KMSKS loop rearrangement for amino acid activation by bacterial tyrosyl-tRNA synthetase. J. Mol. Biol. 346, 105-117 (2005)

28. Kuratani, M. et al. Crystal structures of tyrosyl-tRNA synthetases from Archaea. J. Mol. Biol. 355, 395-408 (2006).

29. Xin, Y., Li, W. \& First, E. A. The 'KMSKS' motif in tyrosyl-tRNA synthetase participates in the initial binding of tRNA(Tyr). Biochemistry 39, 340-347 (2000).

30. Yaremchuk, A., Kriklivyi, I., Tukalo, M. \& Cusack, S. Class I tyrosyl-tRNA synthetase has a class II mode of cognate tRNA recognition. EMBO J. 21, 3829-3840 (2002).

31. Datt, M. \& Sharma, A. Conformational landscapes for KMSKS loop in tyrosyl-tRNA synthetases. J. Struct. Funct. Genomics 15, 45-61 (2014).

32. First, E. A. \& Fersht, A. R. Analysis of the role of the KMSKS loop in the catalytic mechanism of the tyrosyl-tRNA synthetase using multimutant cycles. Biochemistry 34, 5030-5043 (1995).

33. Hountondji, C., Dessen, P. \& Blanquet, S. The SKS of the KMSKS signature of class I aminoacyl-tRNA synthetases corresponds to the GKT/S sequence characteristic of the ATP-binding site of many proteins. Biochimie 75, 1137-1142 (1993)

34. Hountondji, C. et al. Crucial role of conserved lysine 277 in the fidelity of tRNA aminoacylation by Escherichia coli valyl-tRNA synthetase. Biochemistry 41, 14856-14865 (2002)

35. Cusack, S., Yaremchuk, A. \& Tukalo, M. The 2 A crystal structure of leucyl-tRNA synthetase and its complex with a leucyl-adenylate analogue. EMBO J. 19, 2351-2361 (2000).

36. Li, L. et al. Naturally occurring aminoacyl-tRNA synthetases editing-domain mutations that cause mistranslation in Mycoplasma parasites. Proc. Natl Acad. Sci. USA 108, 9378-9383 (2011).

37. Yan, W., Tan, M., Eriani, G. \& Wang, E. D. Leucine-specific domain modulates the aminoacylation and proofreading functional cycle of bacterial leucyl-tRNA synthetase. Nucleic Acids Res. 41, 4988-4998 (2013).
38. Tan, M. et al. The Yin and Yang of tRNA: proper binding of acceptor end determines the catalytic balance of editing and aminoacylation. Nucleic Acids Res. 41, 5513-5523 (2013).

39. Jones, D. A., Ryder, M. H., Clare, B. G., Farrand, S. K. \& Kerr, A. Construction of the Tra- deletion mutant of pAgK84 to safeguard the biological control of crown gall. Mol. Gen. Genet. 212, 207-214 (1988).

40. Clare, B. G., Kerr, A. \& Jones, D. A. Characteristics of the nopaline catabolic plasmid in Agrobacterium strains K84 and K1026 used for biological control of crown gall disease. Plasmid 23, 126-137 (1990).

41. Kovach, M. E. et al. Four new derivatives of the broad-host-range cloning vector pBBR1MCS, carrying different antibiotic-resistance cassettes. Gene 166, 175-176 (1995).

42. Wang, W. \& Malcolm, B. A. Two-stage PCR protocol allowing introduction of multiple mutations, deletions and insertions using QuikChange site-directed mutagenesis. Biotechniques 26, 680-682 (1999).

43. Kim, H. \& Farrand, S. K. Characterization of the acc operon from the nopalinetype Ti plasmid pTiC58, which encodes utilization of agrocinopines A and B and susceptibility to agrocin 84. J. Bacteriol. 179, 7559-7572 (1997).

44. Hayman, G. T. \& Farrand, S. K. Characterization and mapping of the agrocinopine-agrocin 84 locus on the nopaline Ti plasmid pTiC58. J. Bacteriol. 170, 1759-1767 (1988).

45. Hayman, G. T., Beck von Bodman, S., Kim, H., Jiang, P. \& Farrand, S. K. Genetic analysis of the agrocinopine catabolic region of Agrobacterium tumefaciens Ti plasmid pTiC58, which encodes genes required for opine and agrocin 84 transport. J. Bacteriol. 175, 5575-5584 (1993).

46. Wang, Y., Mukhopadhyay, A., Howitz, V. R., Binns, A. N. \& Lynn, D. G. Construction of an efficient expression system for Agrobacterium tumefaciens based on the coliphage T5 promoter. Gene 242, 105-114 (2000).

47. Beck von Bodman, S., Hayman, G. T. \& Farrand, S. K. Opine catabolism and conjugal transfer of the nopaline Ti plasmid pTiC58 are coordinately regulated by a single repressor. Proc. Natl Acad. Sci. USA 89, 643-647 (1992).

48. Kang, Y., Son, M. S. \& Hoang, T. T. One step engineering of T7-expression strains for protein production: increasing the host-range of the T7-expression system. Protein Expr. Purif. 55, 325-333 (2007).

49. Vu, M. T. \& Martinis, S. A. A unique insert of leucyl-tRNA synthetase is required for aminoacylation and not amino acid editing. Biochemistry 46, 5170-5176 (2007).

50. Beebe, K., Waas, W., Druzina, Z., Guo, M. \& Schimmel, P. A universal plate format for increased throughput of assays that monitor multiple aminoacyl transfer RNA synthetase activities. Anal. Biochem. 368, 111-121 (2007).

51. Fersht, A. R. et al. Active site titration and aminoacyl adenylate binding stoichiometry of aminoacyl-tRNA synthetases. Biochemistry 14, 1-4 (1975).

52. Kabsch, W. Xds. Acta Crystallogr. D Biol. Crystallogr. 66, 125-132 (2010).

53. Collaborative Computational Project, N. The CCP4 suite: programs for protein crystallography. Acta Crystallogr. D Biol. Crystallogr. 50, 760-763 (1994).

54. McCoy, A. J., Grosse-Kunstleve, R. W., Storoni, L. C. \& Read, R. J. Likelihood-enhanced fast translation functions. Acta Crystallogr. D Biol. Crystallogr. 61, 458-464 (2005).

55. Arnold, K., Bordoli, L., Kopp, J. \& Schwede, T. The SWISS-MODEL workspace: a web-based environment for protein structure homology modelling. Bioinformatics 22, 195-201 (2006).

56. Tukalo, M., Yaremchuk, A., Fukunaga, R., Yokoyama, S. \& Cusack, S. The crystal structure of leucyl-tRNA synthetase complexed with tRNALeu in the post-transfer-editing conformation. Nat. Struct. Mol. Biol. 12, 923-930 (2005)

57. Emsley, P. \& Cowtan, K. Coot: model-building tools for molecular graphics. Acta Crystallogr. D Biol. Crystallogr. 60, 2126-2132 (2004).

58. Murshudov, G. N., Vagin, A. A. \& Dodson, E. J. Refinement of macromolecular structures by the maximum-likelihood method. Acta Crystallogr. D Biol. Crystallogr. 53, 240-255 (1997).

59. Ginalski, K., Elofsson, A., Fischer, D. \& Rychlewski, L. 3D-Jury: a simple approach to improve protein structure predictions. Bioinformatics 19, 1015-1018 (2003).

60. Luthy, R., Bowie, J. U. \& Eisenberg, D. Assessment of protein models with three-dimensional profiles. Nature 356, 83-85 (1992).

\section{Acknowledgements}

We thank Meighan LeGrand and Dr Ashutosh Tripathy at UNC-CH for their assistance with ITC analysis. We thank Dr Gary Gilleskie and the Biomanufacturing Training and Education Center at North Carolina State University for assistance in large-scale antibiotic preparation and MeadeWestvaco Corporation for their charcoal gift. We thank Prof. David Lynn at Emory University and Dr Tung Hoang at the University of Hawaii for generously providing plasmids and bacterial strains used in this project. We thank members of the ESRF-EMBL Joint Structural Biology Group for access to ESRF beamlines and staff of the EMBL high-throughput crystallization facility, within the Partnership for Structural Biology (PSB). This work was supported by the National Science Foundation [Grant number 1158488 to J.R.]. Funding for open access charge: National Science Foundation [Grant number 1158488 to J.R.] 


\section{Author contributions}

S.Ch. and J.S.R performed project design and the data interpretation, and prepared the manuscript. S.Ch. also conducted the biochemical and biophysical experiments, in vivo mutant constructions and bioassays as well as data analyses. A.P. and S.Cu. solved the structure of the AgnB2 protein ternary complex, provided experimental suggestions and assisted in editing and figures to the final manuscript. C.V. developed the agrobacterial in vivo screen and constructed and assayed initial mutants for this project. S.S. developed AgnB2 and LeuRS $\mathrm{Hp}_{\mathrm{p}}$ protein expression protocols and performed initial biochemical experiments for project. B.T. assisted with structural interpretation of the data and suggestion of mutants to be tested, preparation of figures and assistance in editing manuscript.

\section{Additional information}

Supplementary Information accompanies this paper at http://www.nature.com/ naturecommunications

Competing financial interests: The authors declare no competing financial interests.
Reprints and permission information is available online at http://npg.nature.com/ reprintsandpermissions/

How to cite this article: Chopra, S. et al. Structural characterization of antibiotic self-immunity tRNA synthetase in plant tumour biocontrol agent. Nat. Commun. 7, 12928 doi: 10.1038/ncomms12928 (2016).

\section{(c) (1)}

This work is licensed under a Creative Commons Attribution 4.0 International License. The images or other third party material in this article are included in the article's Creative Commons license, unless indicated otherwise in the credit line; if the material is not included under the Creative Commons license, users will need to obtain permission from the license holder to reproduce the material. To view a copy of this license, visit http://creativecommons.org/licenses/by/4.0/

(C) The Author(s) 2016 